TRANSACTIONS OF THE

AMERICAN MATHEMATICAL SOCIETY

Volume 359, Number 2, February 2007, Pages 489-515

S 0002-9947(06)04326-1

Article electronically published on September 19, 2006

\title{
A HOMOTOPY PRINCIPLE FOR MAPS WITH PRESCRIBED THOM-BOARDMAN SINGULARITIES
}

\author{
YOSHIFUMI ANDO
}

\begin{abstract}
Let $N$ and $P$ be smooth manifolds of dimensions $n$ and $p(n \geq$ $p \geq 2)$ respectively. Let $\Omega^{I}(N, P)$ denote an open subspace of $J^{\infty}(N, P)$ which consists of all Boardman submanifolds $\Sigma^{J}(N, P)$ of symbols $J$ with $J \leq I$. An $\Omega^{I}$-regular map $f: N \rightarrow P$ refers to a smooth map such that $j^{\infty} f(N) \subset \Omega^{I}(N, P)$. We will prove what is called the homotopy principle for $\Omega^{I}$-regular maps on the existence level. Namely, a continuous section $s$ of $\Omega^{I}(N, P)$ over $N$ has an $\Omega^{I}$-regular map $f$ such that $s$ and $j^{\infty} f$ are homotopic as sections.
\end{abstract}

\section{INTRODUCTION}

Let $N$ and $P$ be smooth $\left(C^{\infty}\right)$ manifolds of dimensions $n$ and $p$ respectively with $n \geq p \geq 2$. In $\mathrm{B}$ there have been defined what are called the Boardman manifolds $\Sigma^{I}(N, P)$ in $J^{\infty}(N, P)$ for the symbol $I=\left(i_{1}, i_{2}, \cdots, i_{r}\right)$, where $i_{1}, i_{2}, \cdots, i_{r}$ are a finite number of integers with $i_{1} \geq i_{2} \geq \cdots \geq i_{r} \geq 0$. We say that a smooth map germ $f:(N, x) \rightarrow(P, y)$ has $x$ as a Thom-Boardman singularity of the symbol $I$ if and only if $j_{x}^{\infty} f \in \Sigma^{I}(N, P)$. Let $\Omega^{I}(N, P)$ denote an open subset of $J^{\infty}(N, P)$ which consists of all Boardman manifolds $\Sigma^{J}(N, P)$ with symbols $J$ of length $r$ satisfying $J \leq I$ in the lexicographic order. It is known that $\Omega^{I}(N, P)$ is an open subbundle of $J^{\infty}(N, P)$ with the projection $\pi_{N}^{\infty} \times \pi_{P}^{\infty}$, whose fiber is denoted by $\Omega^{I}(n, p)$. A smooth map $f: N \rightarrow P$ is called an $\Omega^{I}$-regular map if $j^{\infty} f(N) \subset$ $\Omega^{I}(N, P)$.

We will study a homotopy theoretic condition for a given continuous map to be homotopic to an $\Omega^{I}$-regular map. Let $C_{\Omega^{I}}^{\infty}(N, P)$ denote the space consisting of all $\Omega^{I}$-regular maps equipped with the $C^{\infty}$-topology. Let $\Gamma_{\Omega^{I}}(N, P)$ denote the space consisting of all continuous sections of the fiber bundle $\pi_{N}^{\infty} \mid \Omega^{I}(N, P): \Omega^{I}(N, P) \rightarrow$ $N$ equipped with the compact-open topology. Then there exists a continuous map

$$
j_{\Omega^{I}}: C_{\Omega^{I}}^{\infty}(N, P) \rightarrow \Gamma_{\Omega^{I}}(N, P)
$$

defined by $j_{\Omega^{I}}(f)=j^{\infty} f$. It follows from the well-known theorem due to Gromov G1 that if $N$ is a connected open manifold, then $j_{\Omega^{I}}$ is a weak homotopy equivalence. This property is called the homotopy principle (the terminology used in G2]). If $N$ is a closed manifold, then it becomes a hard problem for us to prove the homotopy principle. As the primary investigation preceding [G1, we must refer to the Smale-Hirsch Immersion Theorem ([H1]), $k$-mersion Theorem due to

Received by the editors September 15, 2003.

2000 Mathematics Subject Classification. Primary 58K30; Secondary 57R45, 58A20.

Key words and phrases. Homotopy principle, Thom-Boardman singularity, jet space, Boardman manifold.

(C)2006 American Mathematical Society 
Feit $[\mathrm{F}$ and the Phillips Submersion Theorem for open manifolds ( $[\mathrm{P})$. In [E1] and E2, Èliašberg has proved the well-known homotopy principle on the 1-jet level for $\Omega^{n-p+1,0}$-regular maps, say fold-maps. As for the Thom-Boardman singularities, du Plessis duP has proved that if $i_{r}>n-p-d^{I}$, where $d^{I}$ is the sum of $\alpha_{1}, \cdots, \alpha_{r-1}$ with $\alpha_{\ell}$ being 1 or 0 depending on whether $i_{\ell}-i_{\ell+1}>1$ or otherwise, then $j_{\Omega^{I}}$ is a weak homotopy equivalence.

In this paper we prove the following relative homotopy principle on the existence level for closed manifolds.

Theorem 0.1. Let $n \geq p \geq 2$. Let $N$ and $P$ be connected manifolds of dimensions $n$ and $p$ respectively with $\partial N=\emptyset$. Assume that $\Omega^{I}(N, P)$ contains $\Sigma^{n-p+1,0}(N, P)$ at least. Let $C$ be a closed subset of $N$. Let $s$ be a section in $\Gamma_{\Omega^{I}}(N, P)$ which has an $\Omega^{I}$-regular map $g$ defined on a neighborhood of $C$ to $P$, where $j^{\infty} g=s$.

Then there exists an $\Omega^{I}$-regular map $f: N \rightarrow P$ such that $j^{\infty} f$ is homotopic to $s$ relative to a neighborhood of $C$ by a homotopy $s_{\lambda}$ in $\Gamma_{\Omega^{I}}(N, P)$ with $s_{0}=s$ and $s_{1}=j^{\infty} f$. In particular, we have $f=g$ on a neighborhood of $C$.

In An1 we have given Theorem 0.1 for the symbol $I=(n-p+1, \overbrace{1, \cdots, 1}^{r-1}, 0)$ with a partially sketchy proof using the results in [E1] and [E2]. The singularities of this symbol $I$ are often called $A_{r}$-singularities or Morin singularities. The detailed proof is given in [An4, Theorem 4.1] and An6, Theorem 0.5] for the symbol $I=$ $(n-p+1,0)$. We will use these two theorems in the proof of Theorem 0.1 in this paper.

Recently it turns out that this kind of homotopy principle has many applications. Theorem 0.1 is very important, even for fold-maps, in proving the relations between fold-maps, surgery theory and stable homotopy groups (An4, Theorem 1], An5, Theorems 0.2 and 0.3 ] and [An8]), where the homotopy type of $\Omega^{n-p+1,0}(n, p)$ determined in An3 and An5 has played an important role. We can now readily deduce the famous theorem about the elimination of cusps in [L1] and [E1] (see also [T] ) from these theorems.

The relative homotopy principle on the existence level for maps and singular foliations having only what are called $A, D$ and $E$ singularities is proved in An2 and An7.

In Sady] Sadykov has applied [An1, Theorem 1] to the elimination of higher $A_{r}$ singularities $(r \geq 3)$ for Morin maps when $n-p$ is odd. This result is a strengthened version of the Chess conjecture proposed in [C].

As an application of Theorem 0.1 we prove the following theorem. We note that the simplest case is also a slightly stronger form of the Chess conjecture.

Theorem 0.2. Let $n \geq p \geq 2$, and $N$ and $P$ be connected manifolds of dimensions $n$ and $p$ respectively. Let $I=\left(n-p+1, i_{2}, \cdots, i_{r-1}, 1,1\right)$ and $J=(n-p+1$, $\left.i_{2}, \cdots, i_{r-1}, 1,0\right)$ such that $n-p+1-i_{2}$ and $r(r \geq 3)$ are odd integers. If $f: N \rightarrow P$ is an $\Omega^{I}$-regular map, then $f$ is homotopic to an $\Omega^{J}$-regular map $g: N \rightarrow P$ such that $j^{\infty} f$ and $j^{\infty} g$ are homotopic in $\Gamma_{\Omega^{I}}(N, P)$.

In Section 1 we will explain the notations which are used in this paper. In Section 2 we will review the definitions and the fundamental properties of the Boardman manifolds, from which we deduce several further results about higher intrinsic derivatives in Section 3. In Section 4 we will announce a special form of a homotopy principle in Theorem 4.1 and reduce the proof of Theorem 0.1 to 
the proof of Theorem 4.1 by induction. Furthermore, we will introduce a certain rotation of the tangent spaces defined around the singularities of a given symbol in $N$ for a preliminary deformation of the section $s$. In Section 5 we will prepare several lemmas which are used in the deformation of the section $s$ to the jet extension of an $\Omega^{I}$-regular map in the proof of Theorem 4.1. We will prove Theorem 4.1 in Section 6 , which is the decisive stage in the paper. In Section 7 we will prove Theorem 0.2 by applying Theorem 0.1 .

\section{Notation}

Throughout the paper all manifolds are Hausdorff, paracompact and smooth of class $C^{\infty}$. Maps are basically continuous, but may be smooth (of class $C^{\infty}$ ) if necessary. Given a fiber bundle $\pi: E \rightarrow X$ and a subset $C$ in $X$, we denote $\pi^{-1}(C)$ by $E_{C}$ or $\left.E\right|_{C}$. Let $\pi^{\prime}: F \rightarrow Y$ be another fiber bundle. A map $\tilde{b}: E \rightarrow F$ is called a fiber map over a map $b: X \rightarrow Y$ if $\pi^{\prime} \circ \tilde{b}=b \circ \pi$ holds. The restriction $\tilde{b} \mid E_{C}: E_{C} \rightarrow F$ (or $F_{b(C)}$ ) is denoted by $\tilde{b}_{C}$ or $\left.\tilde{b}\right|_{C}$. We denote, by $b^{F}$, the induced fiber map $b^{*}(F) \rightarrow F$ covering $b$. Let $(b)^{*}\left(\tilde{b} \mid E_{C}\right): E_{C} \rightarrow b^{*} F$ over $C$ for a subset $C \subset X$ and $(b \circ j)^{*}(\tilde{b}): j^{*} E \rightarrow(b \circ j)^{*} F$ over $W$ for a map $j: W \rightarrow X$ be the fiber maps canonically induced from $b$ and $j$ respectively. A fiberwise homomorphism $E \rightarrow F$ is simply called a homomorphism. For a vector bundle $E$ with a metric and a positive function $\delta$ on $X$, let $D_{\delta}(E)$ be the associated disk bundle of $E$ with radius $\delta$. If there is a canonical isomorphism between two vector bundles $E$ and $F$ over $X=Y$, then we write $E \cong F$.

When $E$ and $F$ are smooth vector bundles over $X=Y$, $\operatorname{Hom}(E, F)$ denotes the smooth vector bundle over $X$ with fiber $\operatorname{Hom}\left(E_{x}, F_{x}\right), x \in X$, which consists of all homomorphisms $E_{x} \rightarrow F_{x}$.

Let $J^{k}(N, P)$ denote the $k$-jet space of manifolds $N$ and $P$ ( $k$ may be $\left.\infty\right)$. Let $\pi_{N}^{k}$ and $\pi_{P}^{k}$ be the projections mapping a jet to its source and target respectively. The map $\pi_{N}^{k} \times \pi_{P}^{k}: J^{k}(N, P) \rightarrow N \times P$ induces a structure of a fiber bundle with structure group $L^{k}(p) \times L^{k}(n)$, where $L^{k}(m)$ denotes the group of all $k$-jets of local diffeomorphisms of $\left(\mathbb{R}^{m}, 0\right)$. The fiber $\left(\pi_{N}^{k} \times \pi_{P}^{k}\right)^{-1}(x, y)$ is denoted by $J_{x, y}^{k}(N, P)$.

Let $\pi_{N}$ and $\pi_{P}$ be the projections of $N \times P$ onto $N$ and $P$ respectively. We set

$$
J^{k}(T N, T P)=\bigoplus_{i=1}^{k} \operatorname{Hom}\left(S^{i}\left(\pi_{N}^{*}(T N)\right), \pi_{P}^{*}(T P)\right)
$$

over $N \times P$. Here, for a vector bundle $E$ over $X$, let $S^{i}(E)$ be the vector bundle $\bigcup_{x \in X} S^{i}\left(E_{x}\right)$ over $X$, where $S^{i}\left(E_{x}\right)$ denotes the $i$-fold symmetric product of $E_{x}$. If we provide $N$ and $P$ with Riemannian metrics, then the Levi-Civita connections induce the exponential maps $\exp _{N, x}: T_{x} N \rightarrow N$ and $\exp _{P, y}: T_{y} P \rightarrow P$. In dealing with exponential maps we always consider convex neighborhoods $([\mathrm{K}-\mathrm{N}])$. We define the smooth bundle map

$$
J^{k}(N, P) \rightarrow J^{k}(T N, T P) \quad \text { over } N \times P
$$

by sending $z=j_{x}^{k} f \in J_{x, y}^{k}(N, P)$ to the $k$-jet of $\left(\exp _{P, y}\right)^{-1} \circ f \circ \exp _{N, x}$ at $\mathbf{0} \in$ $T_{x} N$, which is regarded as an element of $J^{k}\left(T_{x} N, T_{y} P\right)\left(=J_{x, y}^{k}(T N, T P)\right)$ (see [K-N], Proposition 8.1] for the smoothness of exponential maps). More strictly, (1.2) gives a smooth equivalence of the fiber bundles under the structure group $L^{k}(p) \times$ $L^{k}(n)$. Namely, it gives a smooth reduction of the structure group $L^{k}(p) \times L^{k}(n)$ of $J^{k}(N, P)$ to $O(p) \times O(n)$, which is the structure group of $J^{k}(T N, T P)$. 
Recall that $S^{i}(E)$ has the inclusion $S^{i}(E) \rightarrow \bigotimes^{i} E$ and the canonical projection $\otimes^{i} E \rightarrow S^{i}(E)$ (see [B, Section 4] and [Mats, Ch. III, Section 2]). Let $E_{j}$ be subbundles of $E(j=1, \cdots, i)$. We define $E_{1} \bigcirc \cdots \bigcirc E_{i}=\bigcirc_{j=1}^{i} E_{j}$ to be the image of $E_{1} \otimes \cdots \otimes E_{i}=\bigotimes_{j=1}^{i} E_{j} \rightarrow \bigotimes^{i} E \rightarrow S^{i}(E)$. When $E_{j+1}=\cdots=E_{j+\ell}$, we often write $E_{1} \bigcirc \cdots \bigcirc E_{j} \bigcirc^{\ell} E_{j+1} \bigcirc E_{j+\ell+1} \bigcirc \cdots \bigcirc E_{i}$ in place of $\bigcirc_{j=1}^{i} E_{j}$.

\section{BOARDMAN MANIFOLDS}

We review well-known results about Boardman manifolds in $J^{\infty}(N, P)$ ( $\mathrm{B}$, [L2] and Math2]). Let $I=\left(i_{1}, \cdots, i_{r}\right)$ be a Boardman symbol with $i_{1} \geq \cdots \geq i_{r} \geq 0$. For $k \leq r$, set $I_{k}=\left(i_{1}, i_{2}, \cdots, i_{k}\right)$ and $\left(I_{k}, 0\right)=\left(i_{1}, i_{2}, \cdots, i_{k}, 0\right)$. In the infinite jet space $J^{\infty}(N, P)$, there has been defined a sequence of submanifolds $\Sigma^{I_{1}}(N, P) \supseteq$ $\cdots \supseteq \Sigma^{I_{r}}(N, P)$ with the following properties. In this paper we often write $\Sigma^{I_{k}}$ for $\Sigma^{I_{k}}(N, P)$ if there is no confusion.

Let $\mathbf{P}=\left(\pi_{P}^{\infty}\right)^{*}(T P)$ and let $\mathbf{D}$ be the total tangent bundle defined over $J^{\infty}(N, P)$. We explain important properties of the total tangent bundle $\mathbf{D}$, which are often used in this paper. Let $f:(N, x) \rightarrow(P, y)$ be a germ and let $\digamma$ be a smooth function in the sense of [B. Definition 1.4] defined on a neighborhood of $j_{x}^{\infty} f$. Given a vector field $v$ defined on a neighborhood of $x$ in $N$, there is a total vector field $D$ defined on a neighborhood of $j_{x}^{\infty} f$ such that $D \digamma \circ j^{\infty} f=v\left(\digamma \circ j^{\infty} f\right)$. It follows that $d\left(j^{\infty} f\right)(v)(\digamma)=D \digamma\left(j^{\infty} f\right)$ for $d\left(j^{\infty} f\right): T N \rightarrow T\left(J^{\infty}(N, P)\right)$ around $x$. This implies $d\left(j^{\infty} f\right)(v)=D$. Since $\pi_{N}^{\infty} \circ j^{\infty} f$ is the identity of $(N, x)$, the differential $d \pi_{N}^{\infty}$ induces the canonical isomorphism

$$
\left(\pi_{N}^{\infty}\right)^{*}\left(d \pi_{N}^{\infty}\right) \mid \mathbf{D}: \mathbf{D} \longrightarrow\left(\pi_{N}^{\infty}\right)^{*}(T N) .
$$

In this paper we often identify $\mathbf{D}$ with $\left(\pi_{N}^{\infty}\right)^{*}(T N)$ by this isomorphism.

First we have the first derivative $\mathbf{d}_{1}: \mathbf{D} \rightarrow \mathbf{P}$ over $J^{\infty}(N, P)$. We define $\Sigma^{I_{1}}(N, P)$ to be the submanifold of $J^{\infty}(N, P)$ which consists of all jets $z$ such that the kernel rank of $\mathbf{d}_{1, z}$ is $i_{1}$. Since $\left.\mathbf{d}_{1}\right|_{\Sigma^{I_{1}}(N, P)}$ is of constant rank $n-i_{1}$, we set $\mathbf{K}_{1}=\operatorname{Ker}\left(\mathbf{d}_{1}\right)$ and $\mathbf{Q}_{1}=\operatorname{Cok}\left(\mathbf{d}_{1}\right)$, which are vector bundles over $\Sigma^{I_{1}}(N, P)$. Set $\mathbf{K}_{0}=\mathbf{D}, \mathbf{P}_{0}=\mathbf{P}$ and $\Sigma^{I_{0}}(N, P)=J^{\infty}(N, P)$. We can inductively define $\Sigma^{I_{k}}(N, P)$ and the bundles $\mathbf{K}_{k}$ and $\mathbf{P}_{k}$ over $\Sigma^{I_{k}}(N, P)(k \geq 1)$ with the following properties:

(1) $\left.\mathbf{K}_{k-1}\right|_{\Sigma^{I_{k}(N, P)}} \supseteq \mathbf{K}_{k}$ over $\Sigma^{I_{k}}(N, P)$.

(2) $\mathbf{K}_{k}$ is an $i_{k}$-dimensional subbundle of $\left.T\left(\Sigma^{I_{k-1}}(N, P)\right)\right|_{\Sigma^{I_{k}(N, P)}}$.

(3) There exists the $(k+1)$-th intrinsic derivative $\mathbf{d}_{k+1}:\left.T\left(\Sigma^{I_{k-1}}(N, P)\right)\right|_{\Sigma^{I_{k}}(N, P)}$ $\rightarrow \mathbf{P}_{k}$ over $\Sigma^{I_{k}}(N, P)$, so that it induces the exact sequence over $\Sigma^{I_{k}}(N, P)$ :

$$
\left.\mathbf{0} \rightarrow T\left(\Sigma^{I_{k}}(N, P)\right) \stackrel{\text { inclusion }}{\hookrightarrow} T\left(\Sigma^{I_{k-1}}(N, P)\right)\right|_{\Sigma^{I_{k}}(N, P)} \stackrel{\mathbf{d}_{k+1}}{\longrightarrow} \mathbf{P}_{k} \rightarrow \mathbf{0} .
$$

Namely, $\mathbf{d}_{k+1}$ induces the isomorphism of the normal bundle

$$
\nu\left(I_{k} \subset I_{k-1}\right)=\left(\left.T\left(\Sigma^{I_{k-1}}(N, P)\right)\right|_{\Sigma^{I_{k}(N, P)}}\right) / T\left(\Sigma^{I_{k}}(N, P)\right)
$$

of $\Sigma^{I_{k}}(N, P)$ in $\Sigma^{I_{k-1}}(N, P)$ onto $\mathbf{P}_{k}$.

(4) $\Sigma^{I_{k+1}}(N, P)$ is defined to be the submanifold of $\Sigma^{I_{k}}(N, P)$ which consists of all jets $z$ with $\operatorname{dim}\left(\operatorname{Ker}\left(\mathbf{d}_{k+1, z} \mid \mathbf{K}_{k, z}\right)\right)=i_{k+1}$. In particular, $\Sigma^{I_{k}}(N, P)$ is the disjoint union $\bigcup_{j=0}^{i_{k}} \Sigma^{\left(I_{k}, j\right)}(N, P)$. 
(5) Set $\mathbf{K}_{k+1}=\operatorname{Ker}\left(\mathbf{d}_{k+1} \mid \mathbf{K}_{k}\right)$ and $\mathbf{Q}_{k+1}=\operatorname{Cok}\left(\mathbf{d}_{k+1} \mid \mathbf{K}_{k}\right)$ over $\Sigma^{I_{k+1}}(N, P)$. Then it follows that $\left.\left(\left.\mathbf{K}_{k}\right|_{\Sigma^{I_{k+1}}(N, P)}\right) \cap T\left(\Sigma^{I_{k}}(N, P)\right)\right|_{\Sigma^{I_{k+1}}(N, P)}=\mathbf{K}_{k+1}$. We have the canonical projection $\mathbf{e}_{k}:\left.\mathbf{P}_{k-1}\right|_{\Sigma^{I}(N, P)} \rightarrow \mathbf{Q}_{k}$.

(6) The intrinsic derivative

$$
d\left(\mathbf{d}_{k+1} \mid \mathbf{K}_{k}\right):\left.T\left(\Sigma^{I_{k}}(N, P)\right)\right|_{\Sigma^{I_{k+1}}(N, P)} \rightarrow \operatorname{Hom}\left(\mathbf{K}_{k+1}, \mathbf{Q}_{k+1}\right) \quad \text { over } \Sigma^{I_{k+1}}(N, P)
$$

of $\mathbf{d}_{k+1} \mid \mathbf{K}_{k}$ is of constant rank $\operatorname{dim}\left(\Sigma^{I_{k}}(N, P)\right)-\operatorname{dim}\left(\Sigma^{I_{k+1}}(N, P)\right)$. We set $\mathbf{P}_{k+1}=$ $\operatorname{Im}\left(d\left(\mathbf{d}_{k+1} \mid \mathbf{K}_{k}\right)\right)$ and define $\mathbf{d}_{k+2}$ to be

$$
\mathbf{d}_{k+2}=d\left(\mathbf{d}_{k+1} \mid \mathbf{K}_{k}\right):\left.T\left(\Sigma^{I_{k}}(N, P)\right)\right|_{\Sigma^{I_{k+1}}(N, P)} \rightarrow \mathbf{P}_{k+1}
$$

as the epimorphism.

(7) There exists a bundle homomorphism of constant rank

$$
\mathbf{u}_{k}: \operatorname{Hom}\left(\mathbf{K}_{k} \bigcirc \mathbf{K}_{k-1} \bigcirc \cdots \bigcirc \mathbf{K}_{1}, \mathbf{P}\right) \rightarrow \operatorname{Hom}\left(\mathbf{K}_{k}, \mathbf{Q}_{k}\right) \quad \text { over } \Sigma^{I_{k}}(N, P)
$$

such that the image of $\mathbf{u}_{k}$ coincides with $\mathbf{P}_{k}$. We denote, by $\mathbf{c}_{k}$, the map $\mathbf{u}_{k}$ as the epimorphism onto $\mathbf{P}_{k}$. Furthermore, $\mathbf{u}_{k}$ is defined as the composition

$$
\begin{aligned}
& \operatorname{Hom}\left(\mathbf{K}_{k} \bigcirc \mathbf{K}_{k-1} \bigcirc \cdots \bigcirc \mathbf{K}_{1}, \mathbf{P}\right) \hookrightarrow \operatorname{Hom}\left(\mathbf{K}_{k} \otimes\left(\mathbf{K}_{k-1} \bigcirc \cdots \bigcirc \mathbf{K}_{1}\right), \mathbf{P}\right) \\
& \cong \operatorname{Hom}\left(\mathbf{K}_{k}, \operatorname{Hom}\left(\mathbf{K}_{k-1} \bigcirc \cdots \bigcirc \mathbf{K}_{1}, \mathbf{P}\right)\right) \stackrel{\operatorname{Hom}\left(i d_{\mathbf{K}_{k}}, \mathbf{c}_{k-1}\right)}{\longrightarrow} \operatorname{Hom}\left(\mathbf{K}_{k}, \mathbf{P}_{k-1}\right) \\
& \stackrel{\operatorname{Hom}\left(i d_{\mathbf{K}_{k}}, \mathbf{e}_{k}\right)}{\longrightarrow} \operatorname{Hom}\left(\mathbf{K}_{k}, \mathbf{Q}_{k}\right)
\end{aligned}
$$

([B, Theorem 7.14]), where $\hookrightarrow$ refers to the inclusion.

(8) For a smooth map $f: N \rightarrow P$ such that $j^{\infty} f$ is transverse to $\Sigma^{I_{k}}(N, P)$, let $S^{I_{k}}\left(j^{\infty} f\right)$ denote $\left(j^{\infty} f\right)^{-1}\left(\Sigma^{I_{k}}(N, P)\right)$. If $f \mid S^{I_{k}}\left(j^{\infty} f\right): S^{I_{k}}\left(j^{\infty} f\right) \rightarrow P$ is of kernel rank $i_{k+1}$ at $x$, then $j_{x}^{\infty} f \in \Sigma^{I_{k+1}}(N, P)$.

(9) The submanifold $\Sigma^{I_{k}}(N, P)$ is actually defined so that it coincides with the inverse image of the submanifold $\widetilde{\Sigma}^{I_{k}}(N, P)$ in $J^{k}(N, P)$ by the canonical projection $\pi_{k}^{\infty}: J^{\infty}(N, P) \rightarrow J^{k}(N, P)$.

(10) The codimension of $\Sigma^{I_{k}}(N, P)$ in $J^{\infty}(N, P)$ is described in $\mathrm{B}$, Theorem 6.1]. In particular, $\operatorname{codim} \Sigma^{I_{k}}(N, P)(k \geq 2)$ is equal to

$$
\begin{aligned}
& \operatorname{codim} \Sigma^{I_{k-1}}(N, P)+\left(p-n+i_{1}\right) \operatorname{dim}\left(\mathbf{K}_{k} \bigcirc \mathbf{K}_{k-1} \bigcirc \cdots \circ \mathbf{K}_{1}\right) \\
& -\left\{\sum_{\ell=2}^{k}\left(i_{\ell-1}-i_{\ell}\right) \operatorname{dim}\left(\mathbf{K}_{k} \bigcirc \mathbf{K}_{k-1} \bigcirc \cdots \circ \mathbf{K}_{\ell}\right)\right\} .
\end{aligned}
$$

We write $I \leq J$ when $\Omega^{I}(n+m, p+m) \subset \Omega^{J}(n+m, p+m)$ for any integer $m$ with $n+m>0$ and $p+m>0$ and write $I<J$ when $I \leq J$ and

$$
\Omega^{I}(n+m, p+m) \subsetneq \Omega^{J}(n+m, p+m)
$$

for some integer $m$ in this paper. In particular, we have $\left(i_{1}, i_{2}, \cdots, i_{r}, 0\right)=$ $\left(i_{1}, i_{2}, \cdots, i_{r}, 0, \cdots, 0\right)$.

Remark 2.1. (1) It is known that $\Omega^{I}(N, P)$ is an open subset of $J^{\infty}(N, P)$ : Let $I=\left(i_{1}, i_{2}, \cdots, i_{r}\right)$. We prove that the closure of $\Sigma^{I}(N, P)$ is contained in the subset which consists of all submanifolds $\Sigma^{J}(N, P)$ with symbol $J$ of length $r$ with $J \geq I$. Let $z \in J^{\infty}(N, P)$ lie in the closure of $\Sigma^{I}(N, P)$. By definition, we first have $\operatorname{dim}\left(\operatorname{Ker}\left(\mathbf{d}_{1, z}\right)\right) \geq i_{1}$. If the symbol of $z$ is $J$ with $J \neq I$, then we can inductively prove that $z$ has a number $k$ such that $\operatorname{dim}\left(\operatorname{Ker}\left(\mathbf{d}_{j, z} \mid \mathbf{K}_{j-1, z}\right)\right)=i_{j}$ for $1 \leq j \leq k<r$ and $\operatorname{dim}\left(\operatorname{Ker}\left(\mathbf{d}_{k+1, z} \mid \mathbf{K}_{k, z}\right)\right)>i_{k+1}$. This implies the assertion. 
(2) If $I=\left(i_{1}, i_{2}, \cdots, i_{r}\right)$ with $r>n, i_{1} \geq n-p+1$ and $\operatorname{codim} \Sigma^{I}(N, P) \leq n$, then it follows from (2.7) that $i_{\ell}=0$ for $\ell>n$.

We show Remark 2.1(2). We set $\mathbf{k}^{k, j}=\mathbf{K}_{k} \bigcirc \mathbf{K}_{k-1} \bigcirc \cdots \bigcirc \mathbf{K}_{j}$. Then by applying (2.7), we have, for $k \geq 2$,

$$
\begin{aligned}
& \operatorname{codim}^{I_{k}}(N, P)-\operatorname{codim} \Sigma^{I_{k-1}}(N, P) \\
& =\left(p-n+i_{1}\right) \operatorname{dim} \mathbf{k}^{k, 1}-\sum_{\ell=2}^{k}\left(i_{\ell-1}-i_{\ell}\right) \operatorname{dim} \mathbf{k}^{k, \ell} \\
& \geq \operatorname{dim} \mathbf{k}^{k, 1}-\sum_{\ell=2}^{k}\left(i_{\ell-1}-i_{\ell}\right) \operatorname{dim} \mathbf{k}^{k, \ell} \\
& =\operatorname{dim} \mathbf{k}^{k, 2} \otimes\left(\mathbf{K}_{1} / \mathbf{K}_{2}\right)+\operatorname{dim} \mathbf{k}^{k, 2} \bigcirc \mathbf{K}_{2}-\sum_{\ell=2}^{k}\left(i_{\ell-1}-i_{\ell}\right) \operatorname{dim} \mathbf{k}^{k, \ell} \\
& =\left(i_{1}-i_{2}\right) \operatorname{dim} \mathbf{k}^{k, 2}+\operatorname{dim} \mathbf{k}^{k, 2} \bigcirc \mathbf{K}_{2}-\sum_{\ell=2}^{k}\left(i_{\ell-1}-i_{\ell}\right) \operatorname{dim} \mathbf{k}^{k, \ell} \\
& \geq \operatorname{dim} \mathbf{k}^{k, 2}-\sum_{\ell=3}^{k}\left(i_{\ell-1}-i_{\ell}\right) \operatorname{dim} \mathbf{k}^{k, \ell} \\
& \geq \operatorname{dim} \mathbf{k}^{k, \ell}-\sum_{j=\ell+1}^{k}\left(i_{j-1}-i_{j}\right) \operatorname{dim} \mathbf{k}^{k, j} \\
& \ldots \ldots \\
& \geq \operatorname{dim}\left(\mathbf{K}_{k} \bigcirc \mathbf{K}_{k-1}\right)-\left(i_{k-1}-i_{k}\right) \operatorname{dim} \mathbf{K}_{k} \\
& \geq \operatorname{dim}\left(\mathbf{K}_{k} \bigcirc \mathbf{K}_{k}\right) \\
& \geq \operatorname{dim} \mathbf{K}_{k}=i_{k} .
\end{aligned}
$$

Hence, if $i_{n+1}>0$, then we have $\operatorname{codim} \Sigma^{I}(N, P)>n$.

\section{Polynomials}

Let $V$ and $W$ be vector spaces over the field $\mathbb{R}$ with inner product of dimensions $v$ and $w$ respectively. Let $e_{1}, e_{2}, \cdots, e_{v}$ and $d_{1}, d_{2}, \cdots, d_{w}$ be orthonormal bases of $V$ and $W$ respectively. We introduce the inner product in $\operatorname{Hom}\left(\otimes^{\ell} V, W\right)$ as follows. Let $h_{i} \in \operatorname{Hom}\left(\otimes^{\ell} V, W\right)(i=1,2)$ and let

$$
h_{1}\left(e_{i_{1}} \otimes \cdots \otimes e_{i_{\ell}}\right)=\sum_{j=1}^{w} a_{i_{1} i_{2} \cdots i_{\ell}}^{j} d_{j} \quad \text { and } \quad h_{2}\left(e_{i_{1}} \otimes \cdots \otimes e_{i_{\ell}}\right)=\sum_{j=1}^{w} b_{i_{1} i_{2} \cdots i_{\ell}}^{j} d_{j} .
$$

Then we define the inner product by

$$
\left\langle h_{1}, h_{2}\right\rangle=\sum_{j=1}^{w}\left(\sum_{i_{1} i_{2} \cdots i_{\ell}} a_{i_{1} i_{2} \cdots i_{\ell}}^{j} b_{i_{1} i_{2} \cdots i_{\ell}}^{j}\right) .
$$

Let $S$ and $T$ be isomorphisms of $V$ and $W$ which preserve the inner products respectively. We define the action of $(T, S)$ on $\operatorname{Hom}\left(\otimes^{\ell} V, W\right)$ by $(T, S) h=T$ 。 $h \circ\left(\bigotimes^{\ell} S^{-1}\right)$. We show by induction on $\ell$ that this inner product is invariant 
with respect to this action. We represent $S^{-1}$ by the matrix $\left(s_{i j}\right)$ under the basis $e_{1}, e_{2}, \cdots, e_{v}$.

The assertion for $\ell=1$ is well known. Assume that the assertion holds for $\ell-1$. Under the canonical isomorphism $\operatorname{Hom}\left(\otimes^{\ell} V, W\right) \cong \operatorname{Hom}\left(V, \operatorname{Hom}\left(\otimes^{\ell-1} V, W\right)\right)$ we let $h \in \operatorname{Hom}\left(\otimes^{\ell} V, W\right)$ correspond to $\bar{h}$, which satisfies $\bar{h}\left(e_{i_{1}}\right)\left(e_{i_{2}} \otimes \cdots \otimes e_{i_{\ell}}\right)=$ $h\left(e_{i_{1}} \otimes e_{i_{2}} \otimes \cdots \otimes e_{i_{\ell}}\right)$. Then we have that $\left\langle h_{1}, h_{2}\right\rangle=\sum_{j=1}^{v}\left\langle\overline{h_{1}}\left(e_{j}\right), \overline{h_{2}}\left(e_{j}\right)\right\rangle$. Hence, we have that

$$
\begin{aligned}
\left\langle(T, S) h_{1},(T, S) h_{2}\right\rangle & =\sum_{i=1}^{v}\left\langle(T, S)\left(\overline{h_{1}}\left(S^{-1}\left(e_{i}\right)\right)\right),(T, S)\left(\overline{h_{2}}\left(S^{-1}\left(e_{i}\right)\right)\right)\right\rangle \\
& =\sum_{i=1}^{v}\left\langle\overline{h_{1}}\left(S^{-1}\left(e_{i}\right)\right), \overline{h_{2}}\left(S^{-1}\left(e_{i}\right)\right)\right\rangle \\
& =\sum_{i=1}^{v}\left\langle\overline{h_{1}}\left(\sum_{j=1}^{v} s_{i j} e_{j}\right), \overline{h_{2}}\left(\sum_{k=1}^{v} s_{i k} e_{k}\right)\right\rangle \\
& =\sum_{i=1}^{v}\left(\sum_{j=1}^{v} s_{i j}\left(\sum_{k=1}^{v} s_{i k}\left\langle\overline{h_{1}}\left(e_{j}\right), \overline{h_{2}}\left(e_{k}\right)\right\rangle\right)\right) \\
& =\sum_{j=1}^{v} \sum_{k=1}^{v}\left(\sum_{i=1}^{v} s_{i j} s_{i k}\right)\left\langle\overline{h_{1}}\left(e_{j}\right), \overline{h_{2}}\left(e_{k}\right)\right\rangle \\
& =\sum_{j=1}^{v} \sum_{k=1}^{v} \delta_{j k}\left\langle\overline{h_{1}}\left(e_{j}\right), \overline{h_{2}}\left(e_{k}\right)\right\rangle \\
& =\sum_{j=1}^{v}\left\langle\overline{h_{1}}\left(e_{j}\right), \overline{h_{2}}\left(e_{j}\right)\right\rangle \\
& =\left\langle h_{1}, h_{2}\right\rangle .
\end{aligned}
$$

We recall that $\operatorname{Hom}\left(\sum_{j=1}^{\ell} \bigcirc^{j} V, W\right)$ is identified with the set of $w$ polynomials of degree $\leq \ell$ having the constant 0 (see Mats, Ch. III, Section 2]). Here, it will be better to use the basis $\partial / \partial x_{1}, \cdots, \partial / \partial x_{v}$ in place of $e_{1}, \cdots, e_{v}$ and write $x_{1}, \cdots, x_{v}$ as their dual basis. Let $\mathbf{V}$ and $\mathbf{W}$ be smooth vector bundles with metric over a manifold $S$ with fibers $V$ and $W$ respectively. Then $\operatorname{Hom}\left(\bigcirc^{\ell} \mathbf{V}, \mathbf{W}\right)$ is also a vector bundle with metric. For a point $c \in S$, take an open neighborhood $U$ around $c$ such that $\left.\mathbf{V}\right|_{U}$ and $\left.\mathbf{W}\right|_{U}$ are the trivial bundles, say $U \times V$ and $U \times W$ respectively. Then an element of $\left.\operatorname{Hom}\left(\bigcirc^{\ell} \mathbf{V}, \mathbf{W}\right)\right|_{U}$ is identified with a polynomial $\sum_{j=1}^{w}\left(\sum_{|\omega|=\ell} A_{\omega}^{j}(c) x_{1}^{\omega_{1}} x_{2}^{\omega_{2}} \cdots x_{v}^{\omega_{v}}\right) d_{j}, c \in U$, where $\omega=\left(\omega_{1}, \omega_{2}, \cdots, \omega_{v}\right), \omega_{i} \geq 0$ $(i=1, \cdots, v)$, and $|\omega|=\omega_{1}+\cdots+\omega_{v}$, and $A_{\omega}^{j}(c)$ is a real number. If $A_{\omega}^{j}(c)$ are smooth functions of $c$, then $\left\{A_{\omega}^{j}(c)\right\}$ defines a smooth section of $\left.\operatorname{Hom}\left(\bigcirc^{\ell} \mathbf{V}, \mathbf{W}\right)\right|_{U}$ over $U$. We have the following lemma under the above notation.

Lemma 3.1. Let $m=\left(m_{1}, \cdots, m_{v}\right)$ with integers $m_{i} \geq 0,|m|=\ell$ and $c \in U$. Then

$$
\begin{aligned}
& \left.\sum_{j}\left(A_{\omega}^{j}(c) x_{1}^{\omega_{1}} x_{2}^{\omega_{2}} \cdots x_{v}^{\omega_{v}}\right) d_{j}\left(\frac{\partial^{m_{1}}}{\partial x_{1}^{m_{1}}} \bigcirc \frac{\partial^{m_{2}}}{\partial x_{2}^{m_{2}}} \bigcirc \cdots \circ \frac{\partial^{m_{v}}}{\partial x_{v}^{m_{v}}}\right)\right|_{c} \\
& = \begin{cases}\mathbf{0} & \text { if } m \neq \omega, \\
\omega_{1} ! \omega_{2} ! \cdots \omega_{v} !\left(\sum_{j} A_{\omega}^{j}(c) d_{j}\right) & \text { if } m=\omega .\end{cases}
\end{aligned}
$$


We now provide $N$ and $P$ with Riemannian metrics respectively. Then they induce metrics on $\mathbf{D}$ and $\mathbf{P}$, and hence induce a metric on

$$
\operatorname{Hom}\left(\mathbf{K}_{k} \bigcirc \mathbf{K}_{k-1} \bigcirc \cdots \bigcirc \mathbf{K}_{1}, \mathbf{P}\right) .
$$

Furthermore, we can prove inductively that $\mathbf{P}_{k}$, and also $\mathbf{Q}_{k+1}$ as the orthogonal complement of $\operatorname{Im}\left(\mathbf{d}_{k+1} \mid \mathbf{K}_{k}\right)$, inherit induced metrics by (6) and (5) in Section 2 respectively. Consequently we have an induced metric on $\operatorname{Hom}\left(\mathbf{K}_{k+1}, \mathbf{Q}_{k+1}\right)$.

Let us recall that $\mathbf{d}_{k} \mid \mathbf{K}_{k-1}: \mathbf{K}_{k-1} \rightarrow \mathbf{P}_{k-1}$ and $\mathbf{e}_{k}: \mathbf{P}_{k-1} \rightarrow \mathbf{Q}_{k}$ over $\Sigma^{I_{k}}(N, P)$ induce the commutative diagram

$$
\begin{aligned}
& \begin{array}{llll}
\mathbf{K}_{k} & \rightarrow \underset{\text { Knclusion } \downarrow}{\mathbf{K}_{k-1}} & \rightarrow & \underset{\mathbf{j}_{\mathbf{Q}_{k}}}{\rightleftarrows}
\end{array} \\
& \mathbf{0} \quad \rightarrow \quad \mathbf{K}_{k-1} / \mathbf{K}_{k} \rightarrow \operatorname{Hom}\left(\mathbf{K}_{k-1}, \mathbf{Q}_{k-1}\right)
\end{aligned}
$$

Since $\mathbf{Q}_{k}$ is the cokernel of $\mathbf{d}_{k} \mid \mathbf{K}_{k-1}$, we obtain the canonical isomorphism

$$
\mathbf{j}_{\mathbf{Q}_{k}}: \mathbf{Q}_{k} \rightarrow \operatorname{Im}\left(\mathbf{d}_{k} \mid \mathbf{K}_{k-1}\right)^{\perp} \quad \text { over } \Sigma^{I_{k}}(N, P),
$$

where the symbol $\perp$ refers to the orthogonal complement. We also use the notation $\mathbf{j}_{\mathbf{Q}_{k}}: \mathbf{Q}_{k} \rightarrow \operatorname{Hom}\left(\mathbf{K}_{k-1}, \mathbf{Q}_{k-1}\right)$.

Let $k \geq 2$. We now construct the homomorphism, for $1 \leq i \leq k$,

$$
\mathbf{q}(k)^{i+1, i+1}:\left.T\left(\Sigma^{I_{i-1}}(N, P)\right)\right|_{\Sigma^{I_{k}(N, P)}} \bigcirc \mathbf{K}_{i} \bigcirc \mathbf{K}_{i-1} \bigcirc \cdots \bigcirc \mathbf{K}_{1} \rightarrow \mathbf{Q}_{1}
$$

over $\Sigma^{I_{k}}(N, P)$ inductively by using $\left.\mathbf{d}_{i+1}\right|_{\Sigma^{I_{k}}(N, P)}:\left.T\left(\Sigma^{I_{i-1}}(N, P)\right)\right|_{\Sigma^{I_{k}(N, P)}} \rightarrow$ $\left.\mathbf{P}_{i}\right|_{\Sigma^{I_{k}(N, P)}}$ as follows. By the inclusion $\left.\left.\mathbf{P}_{i}\right|_{\Sigma^{I_{k}(N, P)}} \subset \operatorname{Hom}\left(\mathbf{K}_{i}, \mathbf{Q}_{i}\right)\right|_{\Sigma^{I_{k}(N, P)}}$ we have the homomorphism

$$
\mathbf{q}(k)_{\otimes}^{i+1,2}:\left(\left.T\left(\Sigma^{I_{i-1}}(N, P)\right)\right|_{\Sigma^{I_{k}}(N, P)}\right) \otimes \mathbf{K}_{i} \rightarrow \mathbf{Q}_{i} \quad \text { over } \Sigma^{I_{k}}(N, P) .
$$

Suppose that we have constructed the homomorphism, for $j \leq i$,

$$
\mathbf{q}(k)_{\otimes}^{i+1, i-j+2}:\left.T\left(\Sigma^{I_{i-1}}(N, P)\right)\right|_{\Sigma^{I_{k}(N, P)}} \otimes \mathbf{K}_{i} \otimes \mathbf{K}_{i-1} \otimes \cdots \otimes \mathbf{K}_{j} \rightarrow \mathbf{Q}_{j}
$$

over $\Sigma^{I_{k}}(N, P)$. By using $\mathbf{j}_{\mathbf{Q}_{j}}: \mathbf{Q}_{j} \rightarrow \operatorname{Hom}\left(\mathbf{K}_{j-1}, \mathbf{Q}_{j-1}\right)$ over $\Sigma^{I_{k}}(N, P)$, we obtain the homomorphism

$$
\mathbf{q}(k)_{\otimes}^{i+1, i-j+3}:\left.T\left(\Sigma^{I_{i-1}}(N, P)\right)\right|_{\Sigma^{I_{k}(N, P)}} \otimes \mathbf{K}_{i} \otimes \mathbf{K}_{i-1} \otimes \cdots \otimes \mathbf{K}_{j-1} \rightarrow \mathbf{Q}_{j-1}
$$

over $\Sigma^{I_{k}}(N, P)$. By setting $j=2$, we obtain $\mathbf{q}(k)_{\otimes}^{i+1, i+1}$. It remains to prove that $\mathbf{q}(k)_{\otimes}^{i+1, i+1}$ is symmetric. This fact has been essentially stated in [B, Section 7, p. 413] without proof. Indeed, the symmetric homomorphism

$$
\mathbf{b}_{i}:\left.T\left(\Sigma^{I_{i-1}}(N, P)\right)\right|_{\Sigma^{I_{i}(N, P)}} \otimes \mathbf{K}_{i} \otimes \mathbf{K}_{i-1} \otimes \cdots \otimes \mathbf{K}_{1} \rightarrow \mathbf{P} \text { over } \Sigma^{I_{i}}(N, P),
$$

has been introduced in $\left[\mathrm{B}\right.$, Section 7, p. 412]. Let $p_{\mathbf{Q}_{1}}^{\mathbf{P}}: \mathbf{P} \rightarrow \mathbf{Q}_{1}$ be the canonical projection. Then $\left.p_{\mathbf{Q}_{1}}^{\mathbf{P}} \circ \mathbf{b}_{i}\right|_{\Sigma^{I_{k}(N, P)}}$ coincides with $\mathbf{q}(k)_{\otimes}^{i+1, i+1}$ by the definition of $\mathbf{b}_{i}$. Note that $\mathbf{q}(k)^{i+1, i+1}$ vanishes on $\left.T\left(\Sigma^{I_{i}}(N, P)\right)\right|_{\Sigma^{I_{k}(N, P)}} \bigcirc \mathbf{K}_{i} \bigcirc \mathbf{K}_{i-1} \bigcirc \cdots \bigcirc \mathbf{K}_{1}$.

Following the proof of $[\mathrm{B}$, Theorem 4.1] we briefly prove the symmetricity. Let $z=j_{x}^{\infty} f \in \Sigma^{I_{k}}(N, P)$. Let $\mathfrak{m}_{y}$ denote the maximal ideal of function germs $(P, y) \rightarrow \mathbb{R}$ vanishing at $y$ and let $\mathfrak{m}_{y}^{\mathbf{Q}}$ denote the ideal of $\mathfrak{m}_{y}$ which consists of all germs $\alpha \in \mathfrak{m}_{y}$ such that the differential $d(\alpha \circ f)$ at $x$ vanishes. Then $\mathbf{Q}_{1, z}$ is canonically identified with $\operatorname{Hom}\left(\mathfrak{m}_{y}^{\mathbf{Q}} /\left(\mathfrak{m}_{y}^{\mathbf{Q}} \cap \mathfrak{m}_{y}{ }^{2}\right), \mathbb{R}\right)$. Let $D$ and $D_{j}$ be sections 
of $\left.T\left(\Sigma^{I_{i-1}}(N, P)\right)\right|_{\Sigma^{I_{k}(N, P)}}$ and $\mathbf{K}_{j}$ respectively defined around $z$ and let $\alpha \in \mathfrak{m}_{y}^{\mathbf{Q}}$. Then (3.1) is regarded as the homomorphism induced from

$$
T\left(\Sigma^{I_{i-1}}(N, P)\right)_{z} \otimes \mathbf{K}_{i, z} \otimes \mathbf{K}_{i-1, z} \otimes \cdots \otimes \mathbf{K}_{1, z} \otimes \mathfrak{m}_{y}^{\mathbf{Q}} /\left(\mathfrak{m}_{y}^{\mathbf{Q}} \cap \mathfrak{m}_{y}^{2}\right) \longrightarrow \mathbb{R}
$$

which maps $D \otimes D_{i} \otimes \cdots \otimes D_{1} \otimes \alpha$ to $\left(D D_{i} \cdots D_{1} \alpha\right)(z)$ (see (a) and (b) in the proof of $[\mathrm{B}$, Theorem 4.1]). We have to show the following for the symmetry (consult Remark 3.2 below to avoid the infinity of the dimensions of the tangent spaces). In the expression with $\left[D_{j}, D_{j-1}\right]=D_{j} D_{j-1}-D_{j-1} D_{j}$

$$
D D_{i} \cdots D_{j} D_{j-1} \cdots D_{1} \alpha-D D_{i} \cdots D_{j-1} D_{j} \cdots D_{1} \alpha=D D_{i} \cdots\left[D_{j}, D_{j-1}\right] \cdots D_{1} \alpha
$$

for some $j$ with $1<j \leq i+1\left(D_{i+1}=D\right)$, we have that $\left[D_{j}, D_{j-1}\right]$ is the section of $\mathbf{K}_{j-1}$ for $j \leq i$ and of $T\left(\Sigma^{I_{i-1}}(N, P)\right)_{z}$ for $j=i+1$ by [B, Lemma 3.2]. Since $\left.\left.\mathbf{K}_{j}\right|_{\Sigma^{I_{k}(N, P)}} \subset \mathbf{K}_{j-1}\right|_{\Sigma^{I_{k}(N, P)}}$, the length of $D D_{i} \cdots\left[D_{j}, D_{j-1}\right] \cdots D_{1}$ is $i, D$ and $\left[D, D_{i}\right]$ lie in $T\left(\Sigma^{I_{i-1}}(N, P)\right)_{z}$ and since $T\left(\Sigma^{I_{i-1}}(N, P)\right)_{z} \subset T\left(\Sigma^{I_{i-2}}(N, P)\right)_{z}$, we have that $\left(D D_{i} \cdots\left[D_{j}, D_{j-1}\right] \cdots D_{1} \alpha\right)(z)=0$ by $\operatorname{Ker}\left(\mathbf{d}_{i, z}\right)=T\left(\Sigma^{I_{i-1}}(N, P)\right)_{z}$ in (2.2). This is what we want.

In particular, if $i=1$ and we restrict $\left.T\left(\Sigma^{I_{i-1}}(N, P)\right)\right|_{\Sigma^{I_{k}}(N, P)}$ to $\mathbf{K}_{1}$, then we have the homomorphism $\mathbf{q}(k)^{2,2} \mid\left(\mathbf{K}_{1} \bigcirc \mathbf{K}_{1}\right): \mathbf{K}_{1} \bigcirc \mathbf{K}_{1} \rightarrow \mathbf{Q}_{1}$ over $\Sigma^{I_{k}}(N, P)$, which induces the nonsingular quadratic form $\left(\mathbf{K}_{1} / \mathbf{K}_{2}\right) \bigcirc\left(\mathbf{K}_{1} / \mathbf{K}_{2}\right) \rightarrow \mathbf{Q}_{1}$ on each fiber.

Remark 3.2. We can entirely do the arguments in Sections 2 and 3 on $J^{\ell}(N, P)$ for a large $\ell$.

We give a proof of Remark 3.2. Take Riemannian metrics on $N$ and $P$ which enable us to consider the exponential maps $T N \rightarrow N$ and $T P \rightarrow P$ by the Levi-Civita connections. For any points $x \in N$ and $y \in P$, we have the normal coordinates systems $\left(x_{1}, \cdots, x_{n}\right)$ and $\left(y_{1}, \cdots, y_{p}\right)$ on convex neighborhoods $U$ of $x$ and $V$ of $y$ associated to orthonormal bases of $T_{x} N$ and $T_{y} P$ respectively (see [K-N]). Let us define the canonical embedding $\mu_{\infty}^{\ell}: J^{\ell}(T N, T P) \rightarrow J^{\infty}(T N, T P)$ by putting the null homomorphism of $\operatorname{Hom}\left(S^{i}\left(\pi_{N}^{*}(T N)\right), \pi_{P}^{*}(T P)\right)$ as the $i$-th component for $i>\ell$. It is clear that $\pi_{\ell}^{\infty} \circ \mu_{\infty}^{\ell}=i d_{J^{\ell}(T N, T P)}$ and $\mu_{\infty}^{\ell} \circ \pi_{\ell}^{\infty} \mid\left(\mu_{\infty}^{\ell}\left(J^{\ell}(T N, T P)\right)\right)=$ $i d_{\mu_{\infty}^{\ell}\left(J^{\ell}(T N, T P)\right)}$. We regard $\mu_{\infty}^{\ell}$ as the map to $J^{\infty}(N, P)$ under the identification $(1.2)$.

We can prove that $\left.\mathbf{D}\right|_{\mu_{\infty}^{\ell}\left(J^{\ell}(T N, T P)\right)}$ is tangent to $\mu_{\infty}^{\ell}\left(J^{\ell}(T N, T P)\right)$. Indeed, let $x \in U \subset N$ and $y \in V \subset P$ be as above. For any points $u \in U$ and $v \in V$, let us consider the bases of $T_{u} U$ and $T_{v} V$ which are induced from the bases of $T_{x} N$ and $T_{y} P$ by the parallel displacements along the geodesics from $x$ to $u$ and $y$ to $v$ determined by the connections respectively. We take the normal coordinate systems $\left(u_{1}, \cdots, u_{n}\right)$ around $u$ in $U$, and $\left(v_{1}, \cdots, v_{p}\right)$ around $v$ in $V$, associated to these bases. We note that $u_{1}, \cdots, u_{n}$ (resp. $v_{1}, \cdots, v_{p}$ ) are smooth functions of $x_{1}, \cdots, x_{n}$ (resp. $\left.y_{1}, \cdots, y_{p}\right)$. Let $\sigma=\left(\sigma_{1}, \cdots, \sigma_{n}\right)$ with nonnegative integers $\sigma_{i}$. We define the coordinate system $X_{i}, Y_{j}$ and $W_{j, \sigma}$ of $J^{\infty}(T U, T V)$ as follows. When $z=j_{u}^{\infty} f$ with $u \in U$ and $v=f(u) \in V$, we set

$$
\begin{aligned}
X_{i} & =x_{i} \circ \pi_{U}^{\infty}, \\
Y_{j} & =y_{j} \circ \pi_{V}^{\infty}, \\
W_{j, \sigma}\left(j_{u}^{\infty} f\right) & =\frac{\partial^{|\sigma|}\left(v_{j} \circ f\right)}{\partial u_{1}^{\sigma_{1}} \cdots \partial u_{n}^{\sigma_{n}}}(u),
\end{aligned}
$$


where $|\sigma| \geq 1$. In this definition we should note that the normal coordinate systems $\left(u_{1}, \cdots, u_{n}\right)$ and $\left(v_{1}, \cdots, v_{p}\right)$ vary depending on points $u$ and $v$.

A smooth function $\Phi$ defined on an open subset of $\mu_{\infty}^{\ell}\left(J^{\ell}(T U, T V)\right)$ is written as $\Phi \circ \mu_{\infty}^{\ell} \circ \pi_{\ell}^{\infty}$ on the same open subset and is a smooth function with variables $X_{i}, Y_{j}$ and $W_{j, \sigma}$ for $1 \leq i \leq n, 1 \leq j \leq p$ and $1 \leq|\sigma| \leq \ell$. Hence, $\Phi$ is extended to $\left(\Phi \circ \mu_{\infty}^{\ell}\right) \circ \pi_{\ell}^{\infty}$ defined on an open subset of $J^{\infty}(T U, T V)$, which is a smooth function in the sense of [B, Definition (1.4)]. By using [B, (1.8)], we have that $D_{i}(\Phi)(z)$ is equal to

$$
\begin{aligned}
& \frac{\partial\left(\Phi \circ j^{\infty} f\right)}{\partial x_{i}}(u) \\
& =\frac{\partial \Phi}{\partial X_{i}}(z) \frac{\partial\left(X_{i} \circ j^{\infty} f\right)}{\partial x_{i}}(u)+\sum_{j} \frac{\partial \Phi}{\partial Y_{j}}(z) \frac{\partial\left(Y_{j} \circ j^{\infty} f\right)}{\partial x_{i}}(u) \\
& +\sum_{j, \sigma} \frac{\partial \Phi}{\partial W_{j, \sigma}}(z) \frac{\partial\left(W_{j, \sigma} \circ j^{\infty} f\right)}{\partial x_{i}}(u) \\
& =\frac{\partial \Phi}{\partial X_{i}}(z) \frac{\partial x_{i}}{\partial x_{i}}(u)+\sum_{j, k, h} \frac{\partial \Phi}{\partial Y_{j}}(z) \frac{\partial y_{j}}{\partial v_{k}}(f(u))\left(\frac{\partial\left(v_{k} \circ f\right)}{\partial u_{h}}(u) \frac{\partial u_{h}}{\partial x_{i}}(u)\right) \\
& +\sum_{j, \sigma} \frac{\partial \Phi}{\partial W_{j, \sigma}}(z) \sum_{h}\left(\frac{\partial\left(W_{j, \sigma} \circ j^{\infty} f\right)}{\partial u_{h}}(u) \frac{\partial u_{h}}{\partial x_{i}}(u)\right) \\
& =\frac{\partial \Phi}{\partial X_{i}}(z)+\sum_{j, k, h} \frac{\partial \Phi}{\partial Y_{j}}(z) \frac{\partial y_{j}}{\partial v_{k}}\left(\pi_{P}^{\infty}(z)\right) W_{k, \overline{e_{h}}}(z) \frac{\partial u_{h}}{\partial x_{i}}\left(\pi_{N}^{\infty}(z)\right) \\
& +\sum_{j, \sigma} \frac{\partial \Phi}{\partial W_{j, \sigma}}(z)\left(\sum_{h} W_{j, \sigma^{\prime}}(z) \frac{\partial u_{h}}{\partial x_{i}}\left(\pi_{N}^{\infty}(z)\right)\right),
\end{aligned}
$$

where

(i) for each $h$ and $i$, the function $\partial u_{h} / \partial x_{i}$ is a smooth function of $x_{1}, \cdots, x_{n}$,

(ii) for each $j$ and $k$, the function $\partial v_{k} / \partial y_{j}$ is a smooth function of $y_{1}, \cdots, y_{p}$, and hence $\partial y_{j} / \partial v_{k}$ is also a smooth function of $y_{1}, \cdots, y_{p}$,

(iii) $\overline{e_{h}}=(0, \cdots, 0,1,0, \cdots, 0)$, where the $h$-th component is equal to 1 ,

(iv) setting $\sigma^{\prime}=\left(\sigma_{1}, \cdots, \sigma_{h-1}, \sigma_{h}+1, \sigma_{h+1}, \cdots, \sigma_{n}\right)$ we have $\left(\partial\left(W_{j, \sigma} \circ j^{\infty} f\right) / \partial u_{h}\right)(u)=\partial / \partial u_{h}\left(\partial^{|\sigma|}\left(v_{j} \circ f\right) / \partial u_{1}^{\sigma_{1}} \cdots \partial u_{n}^{\sigma_{n}}\right)(u)=W_{j, \sigma^{\prime}}\left(j_{u}^{\infty} f\right)$,

(v) $|\sigma| \leq \ell-1$, since $W_{j, \sigma^{\prime}}(z)$ vanishes for $|\sigma| \geq \ell$.

Since $\Phi$ is a function of variables $X_{i}, Y_{j}$ and $W_{j, \sigma}$ with $0 \leq|\sigma| \leq \ell$, so is $D_{i}(\Phi)$. Therefore, $D_{i}$ is tangent to $\mu_{\infty}^{\ell}\left(J^{\ell}(T N, T P)\right)$ at $z$ for each $i$. Since $\mathbf{D}$ is locally generated by $D_{i}, \mathbf{D}_{z}$ is tangent to $\mu_{\infty}^{\ell}\left(J^{\ell}(T N, T P)\right)$ at $z$. It suffices for Remark 3.2 to note that we can do the arguments in Sections 2 and 3 just on $\mu_{\infty}^{\ell}\left(J^{\ell}(T N, T P)\right)$.

\section{Primary obstruction}

Let $\mathfrak{s} \in \Gamma_{\Omega^{I}}(N, P)$ be smooth around $\mathfrak{s}^{-1}\left(\Sigma^{J}(N, P)\right)$ and transverse to $\Sigma^{J}(N, P)$. We set $S^{J}(\mathfrak{s})=\mathfrak{s}^{-1}\left(\Sigma^{J}(N, P)\right),\left(\mathfrak{s} \mid S^{J}(\mathfrak{s})\right)^{*}\left(\mathbf{K}_{j}\right)=K_{j}\left(S^{J}(\mathfrak{s})\right),\left(\mathfrak{s} \mid S^{J}(\mathfrak{s})\right)^{*} \mathbf{Q}_{1}=$ $Q\left(S^{J}(\mathfrak{s})\right)$ and $\left(\mathfrak{s} \mid S^{J}(\mathfrak{s})\right)^{*}\left(\mathbf{P}_{j}\right)=P_{j}\left(S^{J}(\mathfrak{s})\right)$. We often write $S^{J}(\mathfrak{s})$ as $S^{J}$ if there is no confusion. Set $\Sigma^{I}(n, p)=\Sigma^{I}\left(\mathbb{R}^{n}, \mathbb{R}^{p}\right) \cap J_{0,0}^{\infty}\left(\mathbb{R}^{n}, \mathbb{R}^{p}\right)$ and $\Omega^{I}(n, p)=\Omega^{I}\left(\mathbb{R}^{n}, \mathbb{R}^{p}\right) \cap$ $J_{0,0}^{\infty}\left(\mathbb{R}^{n}, \mathbb{R}^{p}\right)$.

Let $L=\left(\ell_{1}, \ell_{2}, \cdots, \ell_{n}, 0\right)$ and $\operatorname{codim} \Sigma^{L}(n, p) \leq n$. Let $\Gamma_{\Omega^{L}}^{t r}(N, P)$ denote the subspace of $\Gamma_{\Omega^{L}}(N, P)$ consisting of all smooth sections of $\pi_{N}^{\infty} \mid \Omega^{L}(N, P)$ : 
$\Omega^{L}(N, P) \rightarrow N$ which are transverse to $\Sigma^{J}(N, P)$ for every symbol $J$. Here, $J$ is of length $\leq n+1$ by Remark 2.1(2).

Let $I=\left(i_{1}, i_{2}, \cdots, i_{k}, 0\right)$, where $I \leq L, i_{k}>0$, and $\operatorname{codim} \Sigma^{I}(n, p) \leq n$. Let $C\left(I^{+}\right)$(resp. $\left.C(I)\right)$ refer to the union $C \cup\left(\bigcup_{J>I} S^{J}(s)\right)$ (resp. $\left.C \cup\left(\bigcup_{J \geq I} S^{J}(s)\right)\right)$ where $C$ is a closed subset of $N$ and $\operatorname{codim} \Sigma^{J}(n, p) \leq n$. Here, $s \in \Gamma_{\Omega^{L}}(N, P)$ is, of course, assumed to be smooth around $C\left(I^{+}\right)$(resp. $C(I)$ ) and to be transverse to $\Sigma^{J}(N, P)$ for all symbols $J>I$ (resp. $J \geq I$ ). We often regard $I$ as the symbol $\left(i_{1}, i_{2}, \cdots, i_{k}, 0, \cdots, 0\right)$ of length $n+1$.

We show in this section that it is enough for the proof of Theorem 0.1 to prove the following theorem.

Theorem 4.1. Let $N$ and $P$ be connected manifolds of dimensions $n$ and $p$ respectively with $\partial N=\emptyset$ and $n \geq p \geq 2$. Let $L=\left(\ell_{1}, \ell_{2}, \cdots, \ell_{n}, 0\right), I=\left(i_{1}, i_{2}, \cdots, i_{k}, 0\right)$, $C\left(I^{+}\right)$and $C(I)$ be as above. Assume that $\Omega^{L}(N, P)$ contains $\Sigma^{n-p+1,0}(N, P)$ at least. Let $s$ be a section in $\Gamma_{\Omega^{L}}^{t r}(N, P)$ which has an $\Omega^{L}$-regular map $g\left(I^{+}\right)$defined on a neighborhood of $\left.\mathrm{C} \mathrm{I}^{+}\right)$to $P$, where $j^{\infty} \mathrm{g}\left(\mathrm{I}^{+}\right)=s$. Then there exists a homotopy $s_{\lambda} \in \Gamma_{\Omega^{L}}(N, P)$ of $s_{0}=s$ relative to a neighborhood of $C\left(I^{+}\right)$which satisfies the following properties:

(4.1.1) $s_{1} \in \Gamma_{\Omega^{L}}^{t r}(N, P)$ and if $I>(n-p+1,0)$, then $s_{1}(N \backslash C(I))$ is contained in $\Omega^{I}(N, P) \backslash \Sigma^{I}(N, P)$.

(4.1.2) There exists an $\Omega^{L}$-regular map $g_{I}$ defined on a neighborhood of $C(I)$ (resp. on $N$ when $I=(n-p+1,0))$, where $j^{\infty} g_{I}=s_{1}$ holds. In particular, $g_{I}=g\left(I^{+}\right)$on a neighborhood of $C\left(I^{+}\right)$.

The case $I=(n-p+1,0)$ of Theorem 4.1 follows from Theorem 1 of An1, where a partially sketchy proof was given, and the detailed proof was given in An4, Theorem 4.1] and [An6, Theorem 0.5] in which [E1, 2.2 Theorem] and [E2, Theorem 4.7] have played important roles.

Proof of Theorem 0.1. Take an open neighborhood $U(C)$ of $C$ where the given $\Omega^{I}$ regular map $g$ is defined. Let $U_{i}(C)(i=1,2,3,4,5)$ be open neighborhoods of $C$ with $U_{5}(C)=U(C)$ such that $\mathrm{Cl}_{i}(C) \subset U_{i+1}(C)(i=1,2,3,4)$. Here, "Cl" refers to the topological closure. By [G-G, Ch. II, Corollary 4.11] there exists a homotopy of $\Omega^{I}$-regular maps $g_{\lambda}: U_{5}(C) \rightarrow P$ relative to $\mathrm{Cl}_{1}(C)$ such that $g_{0}=g$ and $j^{\infty} g_{1} \mid U_{5}(C) \backslash U_{2}(C)$ is transverse to $\Sigma^{J}(N, P)$ for all symbols $J$. By applying the homotopy extension property we obtain a homotopy $\mu_{\lambda}$ in $\Gamma_{\Omega^{I}}(N, P)$ such that $\mu_{0}=s, \mu_{\lambda}\left|\mathrm{Cl}_{4}(C)=j^{\infty} g_{\lambda}\right| \mathrm{Cl}_{4}(C)$ and $\mu_{1} \mid\left(N \backslash \mathrm{Cl}_{2}(C)\right) \in \Gamma_{\Omega^{I}}^{t r}\left(N \backslash \mathrm{Cl} U_{2}(C), P\right)$.

Let $N^{\prime}=N \backslash \mathrm{Cl} U_{2}(C), C^{\prime}=\mathrm{Cl}_{3}(C) \cap N^{\prime}$ and $g^{\prime}=g_{1} \mid\left(U_{4}(C) \backslash \mathrm{Cl} U_{2}(C)\right)$. By Section $2(4)$ and Remark 2.1(2) there exists a smallest symbol $L=\left(\ell_{1}, \cdots, \ell_{n}, 0\right)$ such that $\Omega^{L}(n, p) \subset \Omega^{I}(n, p), \operatorname{codim} \Sigma^{L}(N, P) \leq n$ and $\mu_{1} \mid N^{\prime} \in \Gamma_{\Omega^{L}}^{t r}\left(N^{\prime}, P\right)$. We apply Theorem 4.1 to the case of $\mu_{1} \mid N^{\prime}, C^{\prime}, g^{\prime}$ and $J^{\infty}\left(N^{\prime}, P\right)$. By Remark 2.1(2), we can choose a symbol $J=\left(j_{1}, j_{2}, \cdots, j_{k}, 0\right)$ which is the largest symbol of length $n+1$ such that $J \leq L, S^{J}\left(\mu_{1}\right) \backslash C^{\prime} \neq \emptyset$ and $\operatorname{codim} \Sigma^{J}(n, p) \leq n$. Then we first set $C\left(J^{+}\right)=C^{\prime}$ and $g\left(J^{+}\right)=g^{\prime}$. By Theorem 4.1 there exist a homotopy $s_{\lambda}^{\prime}$ in $\Gamma_{\Omega^{L}}\left(N^{\prime}, P\right)$ relative to a neighborhood of $C^{\prime}$ with $s_{0}^{\prime}=\mu_{1} \mid N^{\prime}$ and $s_{1}^{\prime} \in \Gamma_{\Omega^{L}}^{t r}\left(N^{\prime}, P\right)$ and an $\Omega^{L}$-regular map $g_{J}^{\prime}$ defined on a neighborhood of $C(J)$ in $N^{\prime}$, where $j^{\infty} g_{J}^{\prime}=$ $s_{1}^{\prime}$ holds. Then we can prove by downward induction on the symbols that there exists an $\Omega^{L}$-regular map $f^{\prime}: N^{\prime} \rightarrow P$ such that $j^{\infty} f^{\prime}$ is homotopic to $s_{1}^{\prime}$ relative to a neighborhood of $\mathrm{Cl}_{3}(C) \backslash \mathrm{Cl} U_{2}(C)$ by a homotopy $s_{\lambda}^{\prime \prime}$ in $\Gamma_{\Omega^{L}}\left(N^{\prime}, P\right)$ with $s_{0}^{\prime \prime}=s_{1}^{\prime}$ and $s_{1}^{\prime \prime}=j^{\infty} f^{\prime}$. Now we have the homotopy $\bar{\mu}_{\lambda}$ in $\Gamma_{\Omega^{I}}(N, P)$ defined by $\bar{\mu}_{\lambda} \mid N^{\prime}=s_{2 \lambda}^{\prime}$ 
$(0 \leq \lambda \leq 1 / 2), \bar{\mu}_{\lambda} \mid N^{\prime}=s_{2 \lambda-1}^{\prime \prime}(1 / 2 \leq \lambda \leq 1)$ and $\bar{\mu}_{\lambda}\left|\mathrm{Cl} U_{3}(C)=j^{\infty} g_{1}\right| \mathrm{Cl}_{3}(C)$. Thus we obtain the required homotopy $s_{\lambda}$ in Theorem 0.1 by pasting $\mu_{\lambda}$ and $\bar{\mu}_{\lambda}$.

In the rest of Section 4 and in Sections 5 and 6 we use the notation $\Omega$ for $\Omega^{L}$ in Theorem 4.1.

We begin by preparing several notions and results, which are necessary for the proof of Theorem 4.1. For the map $g\left(I^{+}\right)$, we take an open neighborhood $U\left(C\left(I^{+}\right)\right)^{\prime}$ of $C\left(I^{+}\right)$where $g\left(I^{+}\right)$is defined and $j^{\infty} g\left(I^{+}\right)=s$. Without loss of generality we may assume that $N \backslash U\left(C\left(I^{+}\right)\right)^{\prime}$ is nonempty. Take a smooth function $h_{C\left(I^{+}\right)}$: $N \rightarrow[0,1]$ such that

$$
\begin{cases}h_{C\left(I^{+}\right)}(x)=1 & \text { for } x \in C\left(I^{+}\right), \\ h_{C\left(I^{+}\right)}(x)=0 & \text { for } x \in N \backslash U\left(C\left(I^{+}\right)\right)^{\prime}, \\ 0<h_{C\left(I^{+}\right)}(x)<1 & \text { for } x \in U\left(C\left(I^{+}\right)\right)^{\prime} \backslash C\left(I^{+}\right) .\end{cases}
$$

By the Sard Theorem ([H2]) there is a regular value $r$ of $h_{C\left(I^{+}\right)}$with $0<r<$ 1. Then $h_{C\left(I^{+}\right)}^{-1}(r)$ is a submanifold and we set $U\left(C\left(I^{+}\right)\right)=h_{C\left(I^{+}\right)}^{-1}([r, 1])$. We decompose $N \backslash \operatorname{Int} U\left(C\left(I^{+}\right)\right)$to the connected components, say $L_{1}, \cdots, L_{j}, \cdots$. It suffices to prove Theorem 4.1 for each $L_{j} \cup \operatorname{Int} U\left(C\left(I^{+}\right)\right)$. Since $\partial N=\emptyset$, we have that $N \backslash U\left(C\left(I^{+}\right)\right)$has empty boundary. If $L_{j}$ is not compact, then Theorem 4.1 holds for $L_{j} \cup \operatorname{Int} U\left(C\left(I^{+}\right)\right)$by Gromov's theorem (G1, Theorem 4.1.1]). Therefore, it suffices to consider the special case where

(C1) $N \backslash \operatorname{Int} U\left(C\left(I^{+}\right)\right)$is compact, connected and nonempty,

(C2) $\partial U\left(C\left(I^{+}\right)\right)$is a submanifold of dimension $n-1$,

(C3) for the smooth function $h_{C\left(I^{+}\right)}: N \rightarrow[0,1]$ satisfying (4.1) there is a sufficiently small positive real number $\varepsilon$ with $r-2 \varepsilon>0$ such that $r-t \varepsilon(0 \leq t \leq 2)$ are all regular values of $h_{C\left(I^{+}\right)}$.

We have that $h_{C\left(I^{+}\right)}^{-1}([r-2 \varepsilon, 1])$ is contained in $U\left(C\left(I^{+}\right)\right)^{\prime}$. We set $U\left(C\left(I^{+}\right)\right)_{t}=$ $h_{C\left(I^{+}\right)}^{-1}([r-(2-t) \varepsilon, 1])$. In particular, we have $U\left(C\left(I^{+}\right)\right)_{2}=U\left(C\left(I^{+}\right)\right)$. Furthermore, we may assume that

(C4) $s \in \Gamma_{\Omega^{L}}^{t r}(N, P)$, and $S^{I}(s)$ is transverse to $\partial U\left(C\left(I^{+}\right)\right)_{0}$ and $\partial U\left(C\left(I^{+}\right)\right)_{2}$.

Let $\nu\left(\Sigma^{I}\right)$ be the normal bundle $\left(\left.T\left(J^{\infty}(N, P)\right)\right|_{\Sigma^{I}}\right) / T\left(\Sigma^{I}(N, P)\right)$ and let $c(I)=$ $\operatorname{dim} \nu\left(\Sigma^{I}\right)$. Let $\mathbf{j}_{\mathbf{K}}: \mathbf{K}_{1} \rightarrow \nu\left(\Sigma^{I}\right)$ over $\Sigma^{I}(N, P)$ be the composition of the inclusion $\mathbf{K}_{1} \rightarrow T\left(J^{\infty}(N, P)\right)$ and the projection $\left.T\left(J^{\infty}(N, P)\right)\right|_{\Sigma^{I}(N, P)} \rightarrow \nu\left(\Sigma^{I}\right)$. We have the monomorphism

$$
\mathbf{j}_{\mathbf{K}} \circ\left(s \mid S^{I}\right)^{\mathbf{K}_{1}}:\left.K_{1}\left(S^{I}(s)\right) \rightarrow \mathbf{K}_{1}\right|_{\Sigma^{I}(N, P)} \rightarrow \nu\left(\Sigma^{I}\right) .
$$

Let $s \in \Gamma_{\Omega}(N, P)$ be smooth around $s^{-1}\left(\Sigma^{I}(N, P)\right)$ and transverse to $\Sigma^{I}(N, P)$. Let us take a Riemannian metric on $N$. Let $\mathfrak{n}(s, I)$ or simply $\mathfrak{n}(I)$ be the orthogonal normal bundle of $S^{I}(s)$ in $N$. We have the bundle map

$$
d s \mid \mathfrak{n}(s, I): \mathfrak{n}(s, I) \rightarrow \nu\left(\Sigma^{I}\right)
$$

covering $s \mid S^{I}: S^{I}(s) \rightarrow \Sigma^{I}(N, P)$. Let $\mathbf{i}_{\mathfrak{n}(s, I)}:\left.\mathfrak{n}(s, I) \subset T N\right|_{S^{I}}$ denote the inclusion. We define $\Psi(s, I):\left.K_{1}\left(S^{I}(s)\right) \rightarrow \mathfrak{n}(s, I) \subset T N\right|_{S^{I}}$ to be the composition

$$
\begin{aligned}
\mathbf{i}_{\mathfrak{n}(s, I)} \circ & \left(\left(s \mid S^{I}\right)^{*}(d s \mid \mathfrak{n}(s, I))\right)^{-1} \circ\left(\left(s \mid S^{I}\right)^{*}\left(\mathbf{j}_{\mathbf{K}} \circ\left(s \mid S^{I}\right)^{\mathbf{K}_{1}}\right)\right) \\
& :\left.K_{1}\left(S^{I}(s)\right) \longrightarrow\left(s \mid S^{I}\right)^{*} \nu\left(\Sigma^{I}\right) \longrightarrow \mathfrak{n}(s, I) \hookrightarrow T N\right|_{S^{I}} .
\end{aligned}
$$

Let $i_{K_{1}\left(S^{I}(s)\right)}:\left.K_{1}\left(S^{I}(s)\right) \rightarrow T N\right|_{S^{I}}$ be the inclusion. 
Remark 4.2. If $f$ is an $\Omega$-regular map such that $j^{\infty} f$ is transverse to $\Sigma^{I}(N, P)$, then it follows from the definition of $\mathbf{D}$ that $i_{K_{1}\left(S^{I}\left(j^{\infty} f\right)\right)}=\Psi\left(j^{\infty} f, I\right)$ under (2.1).

In what follows let $M=S^{I}(s) \backslash \operatorname{Int}\left(U\left(C\left(I^{+}\right)\right)\right)$. Let $\operatorname{Mono}\left(\left.K_{1}\left(S^{I}(s)\right)\right|_{M},\left.T N\right|_{M}\right)$ denote the subset of $\operatorname{Hom}\left(\left.K_{1}\left(S^{I}(s)\right)\right|_{M},\left.T N\right|_{M}\right)$ which consists of all monomorphisms $K_{1}\left(S^{I}(s)\right)_{c} \rightarrow T_{c} N, c \in M$. We denote the bundle of local coefficients $\mathcal{B}\left(\pi_{j}\left(\operatorname{Mono}\left(K_{1}\left(S^{I}(s)\right)_{c}, T_{c} N\right)\right)\right), c \in M$, by $\mathcal{B}\left(\pi_{j}\right)$, which is a covering space over $M$ with fiber $\pi_{j}\left(\operatorname{Mono}\left(K_{1}\left(S^{I}(s)\right)_{c}, T_{c} N\right)\right)$ defined in [Ste, 30.1]. By the obstruction theory due to [Ste, 36.3], the obstructions for $\left.i_{K_{1}\left(S^{I}(s)\right)}\right|_{M}$ and $\left.\Psi(s, I)\right|_{M}$ to be homotopic relative to $\partial M$ are the primary differences $d\left(\left.i_{K_{1}\left(S^{I}(s)\right)}\right|_{M},\left.\Psi(s, I)\right|_{M}\right)$, which are defined in $H^{j}\left(M, \partial M ; \mathcal{B}\left(\pi_{j}\right)\right)$ with local coefficients. We show that if $I>(n-p+1,0)$, then all of them vanish by Ste, 38.2]. In fact, if $i_{1}=n-p+1$, then we have

$$
\operatorname{dim} M<\operatorname{dim} S^{i_{1}}=n-i_{1}\left(p-n+i_{1}\right)=p-1 .
$$

If $i_{1}>n-p+1$, then

$$
\operatorname{dim} M \leq \operatorname{dim} S^{i_{1}}=n-i_{1}\left(p-n+i_{1}\right)<n-i_{1}<p-1 .
$$

Since $\operatorname{Mono}\left(\mathbb{R}^{i_{1}}, \mathbb{R}^{n}\right)$ is identified with $G L(n) / G L\left(n-i_{1}\right)$, it follows from Ste, 25.6] that $\pi_{j}\left(\operatorname{Mono}\left(\mathbb{R}^{i_{1}}, \mathbb{R}^{n}\right)\right) \cong\{\mathbf{0}\}$ for $j<n-i_{1}(\leq p-1)$. Hence, there exists a homotopy $\psi^{M}(s, I)_{\lambda}:\left.\left.K_{1}\left(S^{I}(s)\right)\right|_{M} \rightarrow T N\right|_{M}$ relative to $M \cap U\left(C\left(I^{+}\right)\right)_{1}$ in $\operatorname{Mono}\left(\left.K_{1}\left(S^{I}(s)\right)\right|_{M},\left.T N\right|_{M}\right)$ such that $\psi^{M}(s, I)_{0}=\left.i_{K_{1}\left(S^{I}(s)\right)}\right|_{M}$ and $\psi^{M}(s, I)_{1}=$ $\left.\Psi(s, I)\right|_{M}$. Let Iso $\left(\left.T N\right|_{M},\left.T N\right|_{M}\right)$ denote the subspace of $\operatorname{Hom}\left(\left.T N\right|_{M},\left.T N\right|_{M}\right)$ which consists of all isomorphisms of $T_{c} N, c \in M$. The restriction map

$$
r_{M}: \operatorname{Iso}\left(\left.T N\right|_{M},\left.T N\right|_{M}\right) \rightarrow \operatorname{Mono}\left(\left.K_{1}\left(S^{I}(s)\right)\right|_{M},\left.T N\right|_{M}\right)
$$

defined by $r_{M}(h)=h \mid\left(K_{1}\left(S^{I}(s)\right)_{c}\right)$, for $h \in \operatorname{Iso}\left(T_{c} N, T_{c} N\right)$, induces a structure of a fiber bundle with fiber Iso $\left(\mathbb{R}^{n-i_{1}}, \mathbb{R}^{n-i_{1}}\right) \times \operatorname{Hom}\left(\mathbb{R}^{n-i_{1}}, \mathbb{R}^{i_{1}}\right)$. By applying the covering homotopy property of the fiber bundle $r_{M}$ to the sections $i d_{\left.T N\right|_{M}}$ and the homotopy $\psi^{M}(s, I)_{\lambda}$, we obtain a homotopy $\Psi(s, I)_{\lambda}:\left.\left.T N\right|_{S^{I}} \rightarrow T N\right|_{S^{I}}$ such that $\Psi(s, I)_{0}=i d_{\left.T N\right|_{S I}},\left.\Psi(s, I)_{\lambda}\right|_{c}=i d_{T_{c} N}$ for all $c \in S^{I} \cap U\left(C\left(I^{+}\right)\right)_{1}$ and $r_{M} \circ \Psi(s, I)_{\lambda} \mid\left(\left.T N\right|_{M}\right)=\psi^{M}(s, I)_{\lambda}$. We define $\Phi(s, I)_{\lambda}:\left.\left.T N\right|_{S^{I}} \rightarrow T N\right|_{S^{I}}$ by $\Phi(s, I)_{\lambda}=\left(\Psi(s, I)_{\lambda}\right)^{-1}$.

\section{LEMMAS}

Let $I$ be the symbol in Theorem 4.1. In the proof of the following lemma, $\left.\Phi(s, I)_{\lambda}\right|_{c}(c \in M)$ is regarded as a linear isomorphism of $T_{c} N$. Let $r_{0}$ be a small positive real number with $r_{0}<1 / 10$. In what follows we set $d_{1}(s, I)=\left(s \mid S^{I}\right)^{*}\left(\mathbf{d}_{1}\right)$.

Lemma 5.1. Let $s \in \Gamma_{\Omega}^{t r}(N, P)$ be a section satisfying the hypotheses of Theorem 4.1. Let $I>(n-p+1,0)$. Then there exists a homotopy $s_{\lambda}$ relative to $U\left(C\left(I^{+}\right)\right)_{2-3 r_{0}}$ in $\Gamma_{\Omega}^{\text {tr }}(N, P)$ with $s_{0}=s$ satisfying

(5.1.1) for any $\lambda, S^{I}\left(s_{\lambda}\right)=S^{I}(s)$ and $\pi_{P}^{\infty} \circ s_{\lambda}\left|S^{I}\left(s_{\lambda}\right)=\pi_{P}^{\infty} \circ s\right| S^{I}(s)$,

(5.1.2) we have $i_{K_{1}\left(S^{I}\left(s_{1}\right)\right)}=\Psi\left(s_{1}, I\right)$, and in particular, $K_{1}\left(S^{I}\left(s_{1}\right)\right)_{c} \subset \mathfrak{n}(s, I)_{c}$ for any point $c \in S^{I}\left(s_{1}\right)$.

Proof. Consider the exponential map $\exp _{N, x}: T_{x} N \rightarrow N$ defined near $\mathbf{0} \in T_{x} N$ by the above Riemannian metric on $N$. We write an element of $\mathfrak{n}(I)_{c}$ as $\mathbf{v}_{c}$. There exists a small positive number $\delta$ such that the map

$$
e:\left.D_{\delta}(\mathfrak{n}(I))\right|_{M} \rightarrow N
$$


defined by $e\left(\mathbf{v}_{c}\right)=\exp _{N, c}\left(\mathbf{v}_{c}\right)$ is an embedding, where $c \in M$ and $\mathbf{v}_{c} \in D_{\delta}\left(\mathfrak{n}(I)_{c}\right)$ (note that $e \mid M$ is the inclusion). Let $\rho:[0, \infty) \rightarrow \mathbb{R}$ be a decreasing smooth function such that $0 \leq \rho(t) \leq 1, \rho(t)=1$ if $t \leq \delta / 10$ and $\rho(t)=0$ if $t \geq \delta$.

Let $\ell(\mathbf{v})$ denote the parallel translation defined by $\ell(\mathbf{v})(\mathbf{a})=\mathbf{a}+\mathbf{v}$. If we represent a jet of $J^{\infty}(N, P)$ by $j_{x}^{\infty} \sigma_{x}$ for a germ $\sigma_{x}:(N, x) \rightarrow(P, y)$, then we define the homotopy $b_{\lambda}: J^{\infty}(N, P) \rightarrow J^{\infty}(N, P)(0 \leq \lambda \leq 1)$ of the bundle maps over $N \times P$ by setting $b_{\lambda}\left(j_{x}^{\infty} \sigma_{x}\right)$ to be

$$
\left\{\begin{aligned}
& j_{x}^{\infty}\left(\left.\sigma_{x} \circ \exp _{N, c} \circ \ell\left(\mathbf{v}_{c}\right) \circ \Phi(s, I)_{\rho\left(\left\|\mathbf{v}_{c}\right\|\right) \lambda}\right|_{c} \circ \ell\left(-\mathbf{v}_{c}\right) \circ \exp _{N, c}^{-1}\right) \\
& j_{x}^{\infty} \sigma_{x} \text { if } x\left(\mathbf{v}_{c}\right), c \in M \text { and }\left\|\mathbf{v}_{c}\right\| \leq \delta, \\
& \text { if } x \notin \operatorname{Im}(e) .
\end{aligned}\right.
$$

If $\delta$ is sufficiently small, then we may suppose that

$$
e\left(\left.D_{\delta}(\mathfrak{n}(I))\right|_{M}\right) \cap U\left(C\left(I^{+}\right)\right)_{2-3 r_{0}} \subset e\left(\left.D_{\delta}(\mathfrak{n}(I))\right|_{M \cap U\left(C\left(I^{+}\right)\right)_{1}}\right) .
$$

If $c \in S^{I} \cap U\left(C\left(I^{+}\right)\right)_{1}$ or if $\left\|\mathbf{v}_{c}\right\| \geq \delta$, then $\left.\Phi(s, I)_{\lambda}\right|_{c}$ or $\left.\Phi(s, I)_{\rho\left(\left\|\mathbf{v}_{c}\right\|\right) \lambda}\right|_{c}$ is equal to $\left.\Phi(s, I)_{0}\right|_{c}=i d_{T_{c} N}$ respectively. Hence, $b_{\lambda}$ is well defined. We define the homotopy $s_{\lambda}$ of $\Gamma_{\Omega}^{t r}(N, P)$ using $b_{\lambda}$ by $s_{\lambda}(x)=b_{\lambda} \circ s(x)$. By (5.1) we have the following:

(1) $\pi_{P}^{\infty} \circ s_{\lambda}(x)=\pi_{P}^{\infty} \circ s(x)$.

(2) Since $b_{\lambda}$ preserves $\Sigma^{J}(N, P)$ for all symbols $J$, the Boardman symbols of $s_{\lambda}(x)$ and $s(x)$ are equal. In particular, we have $S^{I}\left(s_{\lambda}\right)=S^{I}(s)$.

(3) If $c \in S^{I}(s)$, then we have that $\mathfrak{n}(s, I)_{c} \supset K_{1}\left(S^{I}\left(s_{1}\right)\right)_{c}$ and $i_{K_{1}\left(S^{I}\left(s_{1}\right)\right)}=$ $\Psi\left(s_{1}, I\right)$. Indeed, let $\Psi(s, I)_{c}(\mathbf{v})=\mathbf{w}$ with $\mathbf{v} \in K_{1}\left(S^{I}(s)\right)_{c}$ and $\mathbf{w} \in \mathfrak{n}(s, I)_{c}$. Setting $s(c)=j_{c}^{\infty} \sigma_{c}$ we have by (5.1) that

$$
s_{1}(c)=j_{c}^{\infty}\left(\left.\sigma_{c} \circ \exp _{N, c} \circ \Phi(s, I)_{1}\right|_{c} \circ \exp _{N, c}^{-1}\right) .
$$

Since

$$
d_{1}\left(s_{1}, I\right)\left|\left(\Psi(s, I)\left(K_{1}\left(S^{I}(s)\right)_{c}\right)\right)=d_{1}(s, I) \circ \Phi(s, I)_{1}\right|\left(\Psi(s, I)\left(K_{1}\left(S^{I}(s)\right)_{c}\right)\right)
$$

vanishes, we have that $\Psi(s, I)\left(K_{1}\left(S^{I}(s)\right)_{c}\right) \subset K_{1}\left(S^{I}\left(s_{1}\right)\right)_{c}$. By the dimensional reason we have $\Psi(s, I)\left(K_{1}\left(S^{I}(s)\right)_{c}\right)=K_{1}\left(S^{I}\left(s_{1}\right)\right)_{c}$. By $(4.2), \Psi\left(s_{1}, I\right)(\mathbf{w})$ is equal to

$$
\begin{aligned}
& \mathbf{i}_{\mathfrak{n}\left(s_{1}, I\right)} \circ\left(\left(s_{1} \mid S^{I}\right)^{*}\left(d s_{1} \mid \mathfrak{n}\left(s_{1}, I\right)\right)\right)^{-1} \circ\left(\left(s_{1} \mid S^{I}\right)^{*}\left(\mathbf{j}_{\mathbf{K}} \circ\left(s_{1} \mid S^{I}\right)^{\mathbf{K}_{1}}\right)\right)(\mathbf{w}) \\
& =\mathbf{i}_{\mathfrak{n}\left(s_{1}, I\right)} \circ\left(\left(s \mid S^{I}\right)^{*}(d s \mid \mathfrak{n}(s, I))\right)^{-1} \circ\left(\left(s \mid S^{I}\right)^{*}\left(\mathbf{j}_{\mathbf{K}} \circ\left(s \mid S^{I}\right)^{\mathbf{K}_{1}}\right)\right) \circ \Phi(s, I)_{1}(\mathbf{w}) \\
& =\mathbf{i}_{\mathfrak{n}\left(s_{1}, I\right)} \circ \Psi(s, I)(\mathbf{v}) \\
& =\mathbf{w} .
\end{aligned}
$$

(4) $s_{\lambda} \in \Gamma_{\Omega}^{t r}(N, P)$.

Lemma 5.2. Let $s$ be a section in $\Gamma_{\Omega}^{\operatorname{tr}}(N, P)$ satisfying the property (5.1.2) for $s$ (in place of $s_{1}$ ) of Lemma 5.1. Then there exists a homotopy $\alpha_{\lambda}$ relative to $U\left(C\left(I^{+}\right)\right)_{2-3 r_{0}}$ in $\Gamma_{\Omega}(N, P)$ with $\alpha_{0}=s$ such that

(5.2.1) $\alpha_{\lambda}$ is transverse to $\Sigma^{I}(N, P)$ and $S^{I}\left(\alpha_{\lambda}\right)=S^{I}(s)$ for any $\lambda$,

(5.2.2) we have $i_{K_{1}\left(S^{I}\left(\alpha_{1}\right)\right)}=\Psi\left(\alpha_{1}, I\right)$, and in particular, $K_{1}\left(S^{I}\left(\alpha_{1}\right)\right)_{c} \subset \mathfrak{n}(s, I)_{c}$ for any point $c \in S^{I}\left(\alpha_{1}\right)$,

(5.2.3) $\pi_{P}^{\infty} \circ \alpha_{1} \mid S^{I}\left(\alpha_{1}\right)$ is an immersion to $P$ such that

$$
d\left(\pi_{P}^{\infty} \circ \alpha_{1} \mid S^{I}\left(\alpha_{1}\right)\right)=\left(\pi_{P}^{\infty} \circ \alpha_{1}\right)^{T P} \circ d_{1}\left(\alpha_{1}, I\right) \mid T\left(S^{I}\left(\alpha_{1}\right)\right): T\left(S^{I}\left(\alpha_{1}\right)\right) \rightarrow T P,
$$

where $\left(\pi_{P}^{\infty} \circ \alpha_{1}\right)^{T P}:\left(\pi_{P}^{\infty} \circ \alpha_{1}\right)^{*}(T P) \rightarrow T P$ is the canonical induced bundle map. 
Proof. We choose a Riemannian metric of $P$ and identify $Q\left(S^{I}(s)\right)$ with the orthogonal complement of $\operatorname{Im}\left(d_{1}(s, I)\right)$ in $\left(\pi_{P}^{\infty} \circ s \mid S^{I}\right)^{*}(T P)$. Since $\mathbf{K}_{1} \cap T\left(\Sigma^{I}(N, P)\right)=$ $\{\mathbf{0}\}$, it follows that $\left(\pi_{P}^{\infty} \circ s\right)^{T P} \circ d_{1}(s, I) \mid T\left(S^{I}\right)$ is a monomorphism. By the Hirsch Immersion Theorem ([H1, Theorem 5.7]) there exists a smooth homotopy of monomorphisms $m_{\lambda}^{\prime}: T\left(S^{I}\right) \rightarrow T P$ covering a homotopy $m_{\lambda}: S^{I} \rightarrow P$ relative to $U\left(C\left(I^{+}\right)\right)_{2-4 r_{0}}$ such that $m_{0}^{\prime}=\left(\pi_{P}^{\infty} \circ s\right)^{T P} \circ d_{1}(s, I) \mid T\left(S^{I}\right)$ and $m_{1}$ is an immersion with $d\left(m_{1}\right)=m_{1}^{\prime}$. Then we can extend $m_{\lambda}^{\prime}$ to a smooth homotopy $\widetilde{m_{\lambda}^{\prime}}$ : $\left.T N\right|_{S^{I}} \rightarrow T P$ of homomorphisms of constant rank $n-i_{1}$ relative to $U\left(C\left(I^{+}\right)\right)_{2-3 r_{0}}$ so that $\widetilde{m_{0}^{\prime}}=\left(\pi_{P}^{\infty} \circ s\right)^{T P} \circ d_{1}(s, I)$. In fact, let $m: S^{I} \times[0,1] \rightarrow P \times[0,1]$ and let $m^{\prime}: T\left(S^{I}\right) \times[0,1] \rightarrow T P \times[0,1]$ be the maps defined by $m(c, \lambda)=\left(m_{\lambda}(c), \lambda\right)$ and $m^{\prime}(\mathbf{v}, \lambda)=\left(m_{\lambda}^{\prime}(\mathbf{v}), \lambda\right)$ respectively. Let $m^{*}\left(m^{\prime}\right): T\left(S^{I}\right) \times[0,1] \rightarrow m^{*}(T P \times[0,1])$ be the canonical monomorphism induced from $m^{\prime}$ by $m$. Let $\mathcal{F}_{1}=\operatorname{Im}\left(m^{*}\left(m^{\prime}\right)\right)$ and let $\mathcal{F}_{2}$ be the orthogonal complement of $\mathcal{F}_{1}$ in $m^{*}(T P \times[0,1])$. Since $\mathcal{F}_{2}$ is isomorphic to $\left(\left.\mathcal{F}_{2}\right|_{S^{I} \times 0}\right) \times[0,1]$, we obtain a monomorphism of rank $c(I)-i_{1}$

$$
j_{\mathcal{F}}: \operatorname{Im}\left(d_{1}(s, I) \mid \mathfrak{n}(I)\right) \times[0,1] \rightarrow \mathcal{F}_{2} \quad \text { over } S^{I} \times[0,1] .
$$

Since $d_{1}(s, I) \mid\left(\left.T N\right|_{S^{I}}\right)$ is of constant rank $n-i_{1}$, it induces the homomorphism of kernel rank $i_{1}$

$$
d: \mathfrak{n}(I) \times[0,1] \rightarrow \operatorname{Im}\left(d_{1}(s, I) \mid \mathfrak{n}(I)\right) \times[0,1] \stackrel{j_{\mathcal{F}}}{\rightarrow} \mathcal{F}_{2} .
$$

We define $\widetilde{m^{\prime}}$ to be the composition

$$
\begin{gathered}
\left.T N\right|_{S^{I}} \times[0,1] \cong\left(T\left(S^{I}\right) \oplus \mathfrak{n}(I)\right) \times[0,1] \stackrel{m^{*}\left(m^{\prime}\right) \oplus d}{\longrightarrow} \mathcal{F}_{1} \oplus \mathcal{F}_{2} \\
\rightarrow \operatorname{Im}\left(m^{*}\left(m^{\prime}\right)\right) \oplus \operatorname{Cok}\left(m^{*}\left(m^{\prime}\right)\right) \cong m^{*}(T P \times[0,1]) \stackrel{m^{T P \times[0,1]}}{\longrightarrow} T P \times[0,1] .
\end{gathered}
$$

We define $\widetilde{m_{\lambda}^{\prime}}$ by $\left(\widetilde{m_{\lambda}^{\prime}}(\mathbf{v}), \lambda\right)=\widetilde{m^{\prime}}(\mathbf{v}, \lambda)$.

Recall the submanifold $\widetilde{\Sigma}^{i_{1}}(N, P)$ of $J^{1}(N, P)=J^{1}(T N, T P)$ which corresponds to $\Sigma^{i_{1}}(N, P)$ in Section 2, property $(9)$. Then $\pi_{1}^{\infty} \mid \Sigma^{I}(N, P): \Sigma^{I}(N, P) \rightarrow \widetilde{\Sigma}^{i_{1}}(N, P)$ becomes a fiber bundle. We regard $\widetilde{m_{\lambda}^{\prime}}$ as a homotopy $S^{I} \rightarrow \widetilde{\Sigma}^{i_{1}}(N, P)$. By the covering homotopy property to $s \mid S^{I}$ and $\widetilde{m_{\lambda}^{\prime}}$, we obtain a smooth homotopy $\alpha_{\lambda}^{\Sigma}: S^{I} \rightarrow \Sigma^{I}(N, P)$ covering $\widetilde{m_{\lambda}^{\prime}}$ relative to $U\left(C\left(I^{+}\right)\right)_{2-3 r_{0}}$ such that $\alpha_{0}^{\Sigma}=s \mid S^{I}$.

We have a smooth metric of $\mathfrak{n}(I)$ over $S^{I}$. For a sufficiently small positive function $\varepsilon: S^{I} \rightarrow \mathbb{R}$, let $E_{\varepsilon}\left(S^{I}\right)$ denote $\exp _{N} D_{\varepsilon}(\mathfrak{n}(I))$. By using the transversality of $s$ and the homotopy extension property of bundle maps for $s \mid E_{\varepsilon}\left(S^{I}\right)$ and $\alpha_{\lambda}^{\Sigma}$, we first extend $\alpha_{\lambda}^{\Sigma}$ to a smooth homotopy $\beta_{\lambda}$ of $E_{\varepsilon}\left(S^{I}\right)$ to a tubular neighborhood of $\Sigma^{I}(N, P)$, say $U_{\Sigma^{I}}$, covering $\alpha_{\lambda}^{\Sigma}$ relative to $E_{\varepsilon}\left(S^{I}\right) \cap U\left(C\left(I^{+}\right)\right)_{2-3 r_{0}}$ such that $\beta_{0}=$ $s \mid E_{\varepsilon}\left(S^{I}\right)$ and $\beta_{\lambda}$ is transverse to $\Sigma^{I}(N, P)$. Next extend $\beta_{\lambda}$ to a homotopy $\alpha_{\lambda} \in$ $\Gamma_{\Omega}(N, P)$ so that $\alpha_{0}=s, \alpha_{\lambda}\left|E_{\varepsilon}\left(S^{I}\right)=\beta_{\lambda}, \alpha_{\lambda}\right| U\left(C\left(I^{+}\right)\right)_{2-3 r_{0}}=s \mid U\left(C\left(I^{+}\right)\right)_{2-3 r_{0}}$ and that $\alpha_{\lambda}\left(N \backslash\left(\operatorname{Int}\left(E_{\varepsilon}\left(S^{I}\right) \cup U\left(C\left(I^{+}\right)\right)_{2-3 r_{0}}\right)\right) \subset \Omega(N, P) \backslash \operatorname{Int} U_{\Sigma^{I}}\right.$. This is the required homotopy $\alpha_{\lambda}$.

Here we give two lemmas necessary for the proof of Theorem 4.1. Let $\pi: E \rightarrow S$ be a smooth $c(I)$-dimensional vector bundle with a smooth metric over an $(n-c(I))$ dimensional manifold $S$, which is identified with the zero-section. Then we can identify $\exp _{E} \mid D_{\varepsilon}(E)=i d_{D_{\varepsilon}(E)}$.

Lemma 5.3. Let $\pi: E \rightarrow S$ be given as above. Let $f_{m}: E \rightarrow P(m=1,2)$ be $\Omega$-regular maps such that, for any $c \in S$,

(i) $f_{1}\left|S=f_{2}\right| S$, which are immersions and $\left(d f_{1}\right)_{c}=\left(d f_{2}\right)_{c}$, 
(ii) $j^{\infty} f_{m}$ is transverse to $\Sigma^{I}(E, P)$ and $S=S^{I}\left(j^{\infty} f_{1}\right)=S^{I}\left(j^{\infty} f_{2}\right)$,

(iii) $K_{1}\left(S^{I}\left(j^{\infty} f_{1}\right)\right)_{c}=K_{1}\left(S^{I}\left(j^{\infty} f_{2}\right)\right)_{c}$ are tangent to $\pi^{-1}(c)$,

(iv) $K_{j}\left(S^{I}\left(j^{\infty} f_{1}\right)\right)_{c}=K_{j}\left(S^{I}\left(j^{\infty} f_{2}\right)\right)_{c}, P_{j}\left(S^{I}\left(j^{\infty} f_{1}\right)\right)_{c}=P_{j}\left(S^{I}\left(j^{\infty} f_{2}\right)\right)_{c}$, and $T_{c}\left(S^{I_{j-1}}\left(j^{\infty} f_{1}\right)\right)=T_{c}\left(S^{I_{j-1}}\left(j^{\infty} f_{2}\right)\right)$ for $1 \leq j \leq n+1$,

(v) the two homomorphisms $\left(j^{\infty} f_{m} \mid S\right) *\left(\mathbf{d}_{j+1} \circ d\left(j^{\infty} f_{m}\right) \mid T_{c}\left(S^{I_{j-1}}\left(j^{\infty} f_{m}\right)\right)\right)$ of $T_{c}\left(S^{I_{j-1}}\left(j^{\infty} f_{m}\right)\right)$ to $P_{j}\left(S^{I}\left(j^{\infty} f_{m}\right)\right)_{c}$ for $m=1,2$ are equal for $1 \leq j \leq n+1$.

Let $\eta: S \rightarrow[0,1]$ be any smooth function. Let $\varepsilon: S \rightarrow \mathbb{R}$ be a sufficiently small positive smooth function. We define $\mathbf{f}^{\eta}: D_{\varepsilon}(E) \rightarrow P$ by

$$
\mathbf{f}^{\eta}\left(\mathbf{v}_{c}\right)=\exp _{P, f_{1}(c)}\left((1-\eta(c)) \exp _{P, f_{1}(c)}^{-1}\left(f_{1}\left(\mathbf{v}_{c}\right)\right)+\eta(c) \exp _{P, f_{2}(c)}^{-1}\left(f_{2}\left(\mathbf{v}_{c}\right)\right)\right)
$$

for any $\mathbf{v}_{c} \in \pi^{-1}(c)$ with $\left\|\mathbf{v}_{c}\right\| \leq \varepsilon(c)$. Then the map $\mathbf{f}^{\eta}$ is a well-defined $\Omega$-regular map such that for $m=1,2$, and for any $c \in S$,

(5.3.1) $\mathbf{f}^{\eta}\left|S=f_{m}\right| S$ and $\left(d \mathbf{f}^{\eta}\right)_{c}=\left(d f_{m}\right)_{c}$,

(5.3.2) $j^{\infty} \mathbf{f}^{\eta}$ is transverse to $\Sigma^{I}(E, P)$ and $S=S^{I}\left(j^{\infty} \mathbf{f}^{\eta}\right)$,

(5.3.3) $K_{1}\left(S^{I}\left(j^{\infty} \mathbf{f}^{\eta}\right)\right)_{c}$ is tangent to $\pi^{-1}(c)$,

(5.3.4) $K_{j}\left(S^{I}\left(j^{\infty} \mathbf{f}^{\eta}\right)\right)_{c}=K_{j}\left(S^{I}\left(j^{\infty} f_{m}\right)\right)_{c}, P_{j}\left(S^{I}\left(j^{\infty} \mathbf{f}^{\eta}\right)\right)_{c}=P_{j}\left(S^{I}\left(j^{\infty} f_{m}\right)\right)_{c}$ and $T_{c}\left(S^{I_{j-1}}\left(j^{\infty} \mathbf{f}^{\eta}\right)\right)=T_{c}\left(S^{I_{j-1}}\left(j^{\infty} f_{m}\right)\right)$ for $1 \leq j \leq n+1$,

(5.3.5) the homomorphism

$$
\left(j^{\infty} \mathbf{f}^{\eta} \mid S\right)^{*}\left(\mathbf{d}_{j+1} \circ d\left(j^{\infty} \mathbf{f}^{\eta}\right) \mid T_{c}\left(S^{I_{j-1}}\left(j^{\infty} \mathbf{f}^{\eta}\right)\right)\right): T_{c}\left(S^{I_{j-1}}\left(j^{\infty} \mathbf{f}^{\eta}\right)\right) \rightarrow P_{j}\left(S^{I}\left(j^{\infty} \mathbf{f}^{\eta}\right)\right)_{c}
$$

is equal to the homomorphisms $\left(j^{\infty} f_{m} \mid S\right)^{*}\left(\mathbf{d}_{j+1} \circ d\left(j^{\infty} f_{m}\right) \mid T_{c}\left(S^{I_{j-1}}\left(j^{\infty} f_{m}\right)\right)\right)(m=$ $1,2)$ in (v) for $1 \leq j \leq n+1$.

Proof. We take a Riemannian metric on $P$. Then $S$ has the Riemannian metric induced from $f_{1}\left|S=f_{2}\right| S$, which, together with the smooth metric of the vector bundle $E$, yields the Riemannian metric on $E$. In particular, $S$ is a Riemannian submanifold of $E$ and $S$ is orthogonal to each fiber $E_{c}, c \in S$. Then the local coordinates of $\exp _{E, c}\left(K_{1}\left(S^{I}\left(j^{\infty} f_{m}\right)\right)_{c}\right)$ and $\exp _{P, f_{m}(c)}\left(Q\left(S^{I}\left(j^{\infty} f_{m}\right)\right)_{c}\right)$ are independent of coordinates of $S$, where $Q\left(S^{I}\left(j^{\infty} f_{m}\right)\right)_{c}$ is regarded as the orthogonal complement of $\operatorname{Im}\left(d_{1}\left(j^{\infty} f_{m}, I\right)_{c}\right)$ in $T_{f_{m}(c)} P$.

It follows that $\mathbf{f}^{\eta} \mid S$ is an immersion and $\left(d \mathbf{f}^{\eta}\right)_{c}=\left(d f_{m}\right)_{c}$. We prove the assertions (5.3.4) and (5.3.5) for $j=\ell+1$, by induction on $j$, under the inductive assumptions (5.3.4) and (5.3.5) for $j \leq \ell$. We may consider $\eta(c)$ as a constant when dealing with higher intrinsic derivatives by the property of the total tangent bundle $\mathbf{D}$ given in the beginning of Section 2 under (1.2). We have the following exact sequence for all $j(1 \leq j \leq n+1)$ induced from $(2.2)$ for a general section $\mathfrak{s} \in \Gamma_{\Omega}(E, P)$ which is transverse to $\Sigma^{I}(E, P)$,

$$
0 \longrightarrow T_{c}\left(S^{I_{j}}(\mathfrak{s})\right) \longrightarrow T_{c}\left(S^{I_{j-1}}(\mathfrak{s})\right) \stackrel{h}{\longrightarrow} P_{j}\left(S^{I}(\mathfrak{s})\right)_{c} \longrightarrow 0
$$

where $h$ is $\left(\mathfrak{s} \mid S^{I}(\mathfrak{s})\right)^{*}\left(\mathbf{d}_{j+1} \circ d \mathfrak{s} \mid T_{c}\left(S^{I_{j-1}}(\mathfrak{s})\right)\right)$. By comparing the exact sequences (5.2) for $\mathfrak{s}=j^{\infty} \mathbf{f}^{\eta}, j^{\infty} f_{m}$, we have that $T_{c}\left(S^{I_{\ell}}\left(j^{\infty} \mathbf{f}^{\eta}\right)\right)=T_{c}\left(S^{I_{\ell}}\left(j^{\infty} f_{m}\right)\right)$. It follows from (5) in Section 2 and (5.3.5) that $K_{\ell+1}\left(S^{I}\left(j^{\infty} \mathbf{f}^{\eta}\right)\right)_{c}=K_{\ell+1}\left(S^{I}\left(j^{\infty} f_{m}\right)\right)_{c}$ and hence, $P_{\ell+1}\left(S^{I}\left(j^{\infty} \mathbf{f}^{\eta}\right)\right)_{c}=P_{\ell+1}\left(S^{I}\left(j^{\infty} f_{m}\right)\right)_{c}$. Set $K_{j, c}=K_{j}\left(S^{I}(\mathfrak{s})\right)_{c}, P_{j, c}=$ $P_{j}\left(S^{I}(\mathfrak{s})\right)_{c}(j \leq \ell+1)$ and $Q_{c}=Q\left(S^{I}(\mathfrak{s})\right)_{c}$ for $\mathfrak{s}=j^{\infty} \mathbf{f}^{\eta}, j^{\infty} f_{m}$. Let $\mathbf{t} \in T_{c}\left(S^{I_{\ell}}(\mathfrak{s})\right)$ and $\mathbf{v}_{j} \in K_{j, c}$ for $\mathfrak{s}=j^{\infty} \mathbf{f}^{\eta}, j^{\infty} f_{m}$. For a point $f_{m}(c) \in P$, there exists the normal coordinates system $\left(y_{1}, \cdots, y_{p-n+i_{1}}\right)$ associated to $Q_{c}$. It follows from the 
properties of $\mathbf{b}_{\ell+1}$ that setting $\mathbf{w}=\mathbf{t} \bigcirc \mathbf{v}_{\ell+1} \bigcirc \mathbf{v}_{\ell} \bigcirc \cdots \bigcirc \mathbf{v}_{1}$, we have

$$
\begin{aligned}
& p_{\mathbf{Q}_{1}}^{\mathbf{P}} \circ \mathbf{b}_{\ell+1}\left(d\left(j^{\infty} \mathbf{f}^{\eta}\right)(\mathbf{t}) \circ d\left(j^{\infty} \mathbf{f}^{\eta}\right)\left(\mathbf{v}_{\ell+1}\right) \circ \cdots \circ d\left(j^{\infty} \mathbf{f}^{\eta}\right)\left(\mathbf{v}_{1}\right)\right)\left(y_{j} \circ \pi_{P}^{\infty}\right) \\
& =(\mathbf{w})\left(y_{j} \circ \mathbf{f}^{\eta}\right) \\
& =(1-\eta(c))(\mathbf{w})\left(y_{j} \circ f_{1}\right)+\eta(c)(\mathbf{w})\left(y_{j} \circ f_{2}\right) \\
& =(\mathbf{w})\left(y_{j} \circ f_{m}\right) \\
& =p_{\mathbf{Q}_{1}}^{\mathbf{P}} \circ \mathbf{b}_{\ell+1}\left(d\left(j^{\infty} f_{m}\right)(\mathbf{t}) \circ d\left(j^{\infty} f_{m}\right)\left(\mathbf{v}_{\ell+1}\right) \circ \cdots \circ d\left(j^{\infty} f_{m}\right)\left(\mathbf{v}_{1}\right)\right)\left(y_{j} \circ \pi_{P}^{\infty}\right)
\end{aligned}
$$

for $m=1,2$. Therefore, $\left(\mathfrak{s} \mid S^{I}\right)^{*}\left(p_{\mathbf{Q}_{1}}^{\mathbf{p}} \circ \mathbf{b}_{\ell+1}\right) \mid T_{c}\left(S^{I_{\ell}}(\mathfrak{s})\right) \bigcirc K_{\ell+1, c} \bigcirc \cdots \bigcirc K_{1, c}$ for $\mathfrak{s}=j^{\infty} \mathbf{f}^{\eta}, j^{\infty} f_{m}$ coincide with each other, which induce the same homomorphism

$$
\mathfrak{b}_{\ell+1}\left(\mathfrak{s} \mid S^{I}\right): T_{c}\left(S^{I_{\ell}}(\mathfrak{s})\right) \rightarrow \operatorname{Hom}\left(K_{\ell+1, c} \bigcirc K_{\ell, c} \bigcirc \cdots \bigcirc K_{1, c}, Q_{c}\right)
$$

for $\mathfrak{s}=j^{\infty} \mathbf{f}^{\eta}, j^{\infty} f_{m}$. Furthermore, $\mathbf{c}_{\ell+1}$ in (7) of Section 2, with $\mathbf{P}$ being replaced by $\mathbf{Q}_{1}$, induces the same homomorphism

$$
\mathfrak{c}_{\ell+1}\left(\mathfrak{s} \mid S^{I}\right): \operatorname{Hom}\left(K_{\ell+1, c} \bigcirc K_{\ell, c} \bigcirc \cdots \bigcirc K_{1, c}, Q_{c}\right) \rightarrow P_{\ell+1, c}
$$

for $\mathfrak{s}=j^{\infty} \mathbf{f}^{\eta}, j^{\infty} f_{m}$. By [B] Lemmas 7.11 and 7.13 and Definition 7.12] we have

$$
\mathfrak{c}_{\ell+1}\left(\mathfrak{s} \mid S^{I}\right) \circ \mathfrak{b}_{\ell+1}\left(\mathfrak{s} \mid S^{I}\right)=(\mathfrak{s} \mid S)^{*}\left(\mathbf{d}_{\ell+2} \circ d(\mathfrak{s}) \mid T_{c}\left(S^{I_{\ell}}(\mathfrak{s})\right)\right)
$$

for $\mathfrak{s}=j^{\infty} \mathbf{f}^{\eta}, j^{\infty} f_{m}$. Therefore, $\left(j^{\infty} \mathbf{f}^{\eta} \mid S\right)^{*}\left(\mathbf{d}_{\ell+2} \circ d\left(j^{\infty} \mathbf{f}^{\eta}\right) \mid T_{c}\left(S^{I_{\ell}}\left(j^{\infty} \mathbf{f}^{\eta}\right)\right)\right)$ coincides with $\left(j^{\infty} f_{m} \mid S\right)^{*}\left(\mathbf{d}_{\ell+2} \circ d\left(j^{\infty} f_{m}\right) \mid T_{c}\left(S^{I_{\ell}}\left(j^{\infty} f_{m}\right)\right)\right)$. This implies (5.3.5) for $j=\ell+1$. This completes the proof.

The proof of the following lemma is elementary, and so is left to the reader.

Lemma 5.4. Let $\pi: E \rightarrow S$ be given as above. Let $(\Omega, \Sigma)$ be a pair consisting of a manifold and its submanifold of codimension $c(I)$. Let $\varepsilon: S \rightarrow \mathbb{R}$ be a sufficiently small positive smooth function. Let $h: D_{\varepsilon}(E) \rightarrow(\Omega, \Sigma)$ be a smooth map such that $S=h^{-1}(\Sigma)$ and that $h$ is transverse to $\Sigma$. Then there exists a smooth homotopy $h_{\lambda}:\left(D_{\varepsilon}(E), S\right) \rightarrow(\Omega, \Sigma)$ between $h$ and $\exp _{\Omega} \circ d h \mid D_{\varepsilon}(E)$ such that

(5.4.1) $h_{\lambda}\left|S=h_{0}\right| S, S=h_{\lambda}^{-1}(\Sigma)=h_{0}^{-1}(\Sigma)$ for any $\lambda$,

(5.4.2) $h_{\lambda}$ is smooth and is transverse to $\Sigma$ for any $\lambda$,

(5.4.3) $h_{0}=h$ and $h_{1}\left(\mathbf{v}_{c}\right)=\exp _{\Omega, h(c)} \circ d h\left(\mathbf{v}_{c}\right)$ for $c \in S$ and $\mathbf{v}_{c} \in D_{\varepsilon}\left(E_{c}\right)$.

\section{Proof of Theorem 4.1}

In what follows we denote, by $\mu$, the section $\alpha_{1} \in \Gamma_{\Omega}(N, P)$ in Lemma 5.2 which satisfies (5.2.1), (5.2.2) and (5.2.3). We first introduce several homomorphisms between vector bundles over $S^{I}(\mu)$, which are used for the construction of the required $\Omega$-regular map in Theorem 4.1. We take a Riemannian metric on $P$, which induces the Riemannian metric on $S^{I}(\mu)$. Let us choose a Riemannian metric on $N$ which induces a metric of the vector bundle $\mathfrak{n}(I)$ over $S^{I}(\mu)$ such that

(i) $S^{I}(\mu)$ is a Riemannian submanifold,

(ii) for the symbol $I, K_{j}\left(S^{I}(\mu)\right) / K_{j+1}\left(S^{I}(\mu)\right)$ is orthogonal to $S^{I_{j}}(\mu)$ in $S^{I_{j-1}}(\mu)$ for $1 \leq j \leq k$ on $S^{I}(\mu)\left(S^{I_{0}}(\mu)=N\right)$.

Let $1 \leq j \leq k$. Let $Q, K_{j}$ and $P_{j}$ refer to $Q\left(S^{I}(\mu)\right), K_{j}\left(S^{I}(\mu)\right)$ and $P_{j}\left(S^{I}(\mu)\right)$ respectively. Let $\mathfrak{n}\left(\mu, I_{j} \subset I_{j-1}\right)$ or simply $\mathfrak{n}\left(I_{j} \subset I_{j-1}\right)$ be the orthogonal normal bundle of $S^{I_{j}}(\mu)$ in $S^{I_{j-1}}(\mu)$ over $S^{I}(\mu)$. Let $K_{j} / K_{j+1}$ refer to the orthogonal complement of $K_{j+1}$ in $K_{j}$. Then we have $K_{j} / K_{j+1} \subset \mathfrak{n}\left(\mu, I_{j} \subset I_{j-1}\right)$. Let $\mathfrak{n}_{j}^{I}$ 
refer to the orthogonal complement of $K_{j} / K_{j+1}$ in $\mathfrak{n}\left(\mu, I_{j} \subset I_{j-1}\right)$. Now we have the following isomorphism by $(2.2)$

$$
\begin{aligned}
\left(\mu \mid S^{I}\right)^{*}\left(\mathbf{d}_{j+1} \circ d \mu \mid \mathfrak{n}\left(\mu, I_{j}\right.\right. & \left.\left.\subset I_{j-1}\right)\right): \\
\mathfrak{n}\left(\mu, I_{j}\right. & \left.\subset I_{j-1}\right)=K_{j} / K_{j+1} \oplus \mathfrak{n}_{j}^{I} \rightarrow P_{j} .
\end{aligned}
$$

Let us first define the section $\mathfrak{q}^{\prime}(\mu, I)^{1}$ of $\operatorname{Hom}\left(\mathfrak{n}(I), \operatorname{Im}\left(d_{1}(\mu, I) \mid \mathfrak{n}(I)\right)\right)$ over $S^{I}(\mu)$ by $\mathfrak{q}^{\prime}(\mu, I)^{1}=d_{1}(\mu, I) \mid \mathfrak{n}(I)$, which vanishes on $\left.K_{1}\right|_{S^{I}}$ and gives an isomorphism of $\bigoplus_{j=1}^{k} \mathfrak{n}_{j}^{I}$ onto $\operatorname{Im}\left(d_{1}(\mu, I) \mid \mathfrak{n}(I)\right)$.

For $1 \leq j \leq k, \mathbf{q}(k)^{j+1, j+1}$ in (3.1) induces the homomorphism

$$
\mathfrak{q}(\mu, I)^{j+1}: \mathfrak{n}\left(\mu, I_{j} \subset I_{j-1}\right) \bigcirc K_{j} \bigcirc K_{j-1} \bigcirc \cdots \bigcirc K_{1} \rightarrow Q
$$

over $S^{I}(\mu)$ defined as the composition

$$
\left(\left(\mu \mid S^{I}\right)^{*} \mathbf{q}(k)^{j+1, j+1}\right) \circ\left(\left(\left(\mu \mid S^{I}\right)^{*} d \mu \mid \mathfrak{n}\left(\mu, I_{j} \subset I_{j-1}\right)\right) \bigcirc i d_{K_{j}} \bigcirc K_{j-1} \bigcirc \ldots \bigcirc K_{1}\right) .
$$

If we regard $\mathfrak{q}(\mu, I)^{j+1}$ to vanish on the orthogonal complement of $\mathfrak{n}\left(\mu, I_{j} \subset I_{j-1}\right) \bigcirc$ $K_{j} \bigcirc K_{j-1} \bigcirc \cdots \bigcirc K_{1}$, then $\mathfrak{q}(\mu, I)^{j+1}$ defines a section of $\operatorname{Hom}\left(\bigcirc^{j+1} \mathfrak{n}(I), Q\right)$. Then we have the following section of $\operatorname{Hom}\left(\sum_{j=1}^{k} \bigcirc^{j+1} \mathfrak{n}(I), Q\right)$ :

$$
\mathfrak{q}^{\prime}(\mu, I)=\sum_{j=1}^{k} \mathfrak{q}(\mu, I)^{j+1} \quad \text { over } S^{I}(\mu) .
$$

Let us consider the direct sum decompositions

$$
\begin{aligned}
\mathfrak{n}(\mu, I) & =\bigoplus_{j=1}^{k} \mathfrak{n}\left(\mu, I_{j} \subset I_{j-1}\right), \quad \mathfrak{n}\left(\mu, I_{j} \subset I_{j-1}\right)=K_{j} / K_{j+1} \oplus \mathfrak{n}_{j}^{I}, \\
K_{1} & =\bigoplus_{j=1}^{k-1} K_{j} / K_{j+1} \oplus K_{k}, \quad\left(\pi_{P}^{\infty} \circ \mu \mid S^{I}\right)^{*}(T P)=Q \oplus Q^{\perp}
\end{aligned}
$$

and the inclusion $i_{Q}: Q \rightarrow\left(\pi_{P}^{\infty} \circ \mu \mid S^{I}\right)^{*}(T P)$ which is induced by the Riemannian metric on $P$. Let us define the homomorphism $\mathfrak{q}(\mu, I)$ by

$$
\mathfrak{q}(\mu, I)=\left(\pi_{P}^{\infty} \circ \mu \mid S^{I}\right)^{T P} \circ\left(i_{Q} \circ \mathfrak{q}^{\prime}(\mu, I)+\mathfrak{q}^{\prime}(\mu, I)^{1}\right): \sum_{j=1}^{k+1} \bigcirc^{j} \mathfrak{n}(I) \rightarrow T P
$$

covering the immersion $\pi_{P}^{\infty} \circ \mu \mid S^{I}(\mu): S^{I}(\mu) \rightarrow P$.

Let $\left(\partial / \partial x_{1}, \cdots, \partial / \partial x_{c(I)}\right)$ and $\left(\partial / \partial y_{1}, \cdots, \partial / \partial y_{p-n+i_{1}}\right)$ be orthonormal bases of $\mathfrak{n}(I)_{c}$ and $Q_{c}$ for $c \in S^{I}(\mu)$. An element of $\operatorname{Hom}\left(\bigcirc^{j} \mathfrak{n}(I), Q\right)_{c}$ is identified with $\sum_{i=1}^{p-n+i_{1}}\left(\sum_{|\omega|=j} a_{i}^{\omega}(c) x_{1}^{\omega_{1}} x_{2}^{\omega_{2}} \cdots x_{c(I)}^{\omega_{c(I)}}\right) \partial / \partial y_{i}$, where $\omega=\left(\omega_{1}, \omega_{2}, \cdots, \omega_{c(I)}\right), \omega_{\ell} \geq$ $0\left(i=1, \cdots, p-n+i_{1}\right)$, and $|\omega|=\omega_{1}+\cdots+\omega_{c(I)}$ and $a_{i}^{\omega}(c)$ are real numbers. Thus $i_{Q} \circ \mathfrak{q}^{\prime}(\mu, I)+\mathfrak{q}^{\prime}(\mu, I)^{1}$ is regarded as $p-n+i_{1}$ polynomials of $c(I)$ variables $x_{1}, \cdots, x_{c(I)}$ with constant 0 whenever we fix a point $c \in S^{I}(\mu)$.

Proof of Theorem 4.1. We first prove Theorem 4.1 for the case $I>(n-p+1,0)$. Deform $s \in \Gamma_{\Omega}^{t r}(N, P)$ in Theorem 4.1 as above to a section $\mu \in \Gamma_{\Omega}(N, P)$ as in Lemma 5.2 which satisfies (5.2.1), (5.2.2) and (5.2.3) where $\alpha_{1}$ is replaced by $\mu$. Set $S^{I}=S^{I}(\mu), Q=Q\left(S^{I}(\mu)\right), K_{j}=K_{j}\left(S^{I}(\mu)\right)$ and $P_{j}=P_{j}\left(S^{I}(\mu)\right)$. We define $E\left(S^{I}\right)$ to be $\exp _{N}\left(D_{\delta \circ \mu}(\mathfrak{n}(I))\right)$, where $\delta: \Sigma^{I}(N, P) \rightarrow \mathbb{R}$ is a sufficiently small positive function which is constant on $\mu\left(S^{I}(\mu) \backslash \operatorname{Int} U\left(C\left(I^{+}\right)\right)_{2}\right)$. Here, $E\left(S^{I}\right)$ is a tubular neighborhood of $S^{I}$ with the natural projection $\pi_{E}: E\left(S^{I}\right) \rightarrow S^{I}$. 
It suffices for the proof of Theorem 4.1 to prove the following assertion (A). In fact, we obtain a required homotopy $s_{\lambda}$ in Theorem 4.1 by pasting the homotopies $\alpha_{\lambda}$ in Lemma 5.2 and $H_{\lambda}$ in $(\mathbf{A})$.

(A) There exists a homotopy $H_{\lambda}$ relative to $U\left(C\left(I^{+}\right)\right)_{2-r_{0}}$ in $\Gamma_{\Omega}(N, P)$ with $H_{0}=\mu$ satisfying the following:

(1) $H_{\lambda}$ is transverse to $\Sigma^{I}(N, P)$ and $S^{I}\left(H_{\lambda}\right)=S^{I}$ for any $\lambda$.

(2) We have an $\Omega$-regular map $G$ which is defined on a neighborhood of $E\left(S^{I}\right) \cup$ $U\left(C\left(I^{+}\right)\right)_{2-r_{0}}$ to $P$ such that $j^{\infty} G=H_{1}$ on $E\left(S^{I}\right) \cup U\left(C\left(I^{+}\right)\right)_{2-r_{0}}$.

(3) $H_{1} \in \Gamma_{\Omega}^{t r}(N, P)$.

Let us prove $(\mathbf{A})$. Under the identification of $Q$ with the orthogonal $p-n+i_{1}$ dimensional bundle of $\operatorname{Im}\left(d_{1}(\mu, I)\right)$ in $\left(\pi_{P}^{\infty} \circ \mu \mid S^{I}\right)^{*}(T P)$, the map $\exp _{P} \circ$ $\left(\pi_{P}^{\infty} \circ \mu \mid S^{I}\right)^{T P} \mid D_{\gamma}(Q)$ is an immersion for some small positive function $\gamma$. In the proof we express a point of $E\left(S^{I}\right)$ as $\mathbf{v}_{c}$, where $c \in S^{I}, \mathbf{v}_{c} \in \mathfrak{n}(I)_{c}$ and $\left\|\mathbf{v}_{c}\right\| \leq \delta(\mu(c))$. In the proof we say that a smooth homotopy

$$
k_{\lambda}:\left(E\left(S^{I}\right), \partial E\left(S^{I}\right)\right) \rightarrow\left(\Omega(N, P), \Omega(N, P) \backslash \Sigma^{I}(N, P)\right)
$$

has the property $(\mathrm{C})$ if it satisfies for any $\lambda$

$(\mathrm{C}-1) k_{\lambda}^{-1}\left(\Sigma^{I}(N, P)\right)=S^{I}$, and $\pi_{P}^{\infty} \circ k_{\lambda}\left|S^{I}=\pi_{P}^{\infty} \circ k_{0}\right| S^{I}$,

$(\mathrm{C}-2) k_{\lambda}$ is smooth and transverse to $\Sigma^{I}(N, P)$.

If we choose $\delta$ sufficiently small compared with $\gamma$, then we can define the map $g_{0}: E\left(S^{I}\right) \rightarrow P$ by

$$
g_{0}\left(\mathbf{v}_{c}\right)=\exp _{P, \pi_{P}^{\infty} \circ \mu(c)} \circ \mathfrak{q}(\mu, I)_{c} \circ \exp _{N, c}^{-1}\left(\mathbf{v}_{c}\right) .
$$

It follows from Lemma 6.1 below that $g_{0}$ is an $\Omega$-regular map such that $j^{\infty} g_{0}$ is transverse to $\Sigma^{I}(N, P), S^{I}\left(j^{\infty} g_{0}\right)=S^{I}(\mu)$ and $\pi_{P}^{\infty} \circ \mu(c)=g_{0}(c)$ for any $c \in S^{I}$. Now we need to modify $g_{0}$ by using Lemma 5.3 so that $g_{0}$ is compatible with $g\left(I^{+}\right)$.

Let $\eta: S^{I} \rightarrow \mathbb{R}$ be a smooth function such that

(i) $0 \leq \eta(c) \leq 1$ for $c \in S^{I}$,

(ii) $\eta(c)=0$ for $c \in S^{I} \cap U\left(C\left(I^{+}\right)\right)_{2-(7 / 2) r_{0}}$,

(iii) $\eta(c)=1$ for $c \in S^{I} \backslash U\left(C\left(I^{+}\right)\right)_{2-4 r_{0}}$.

Then consider the map $G: E\left(S^{I}\right) \cup U\left(C\left(I^{+}\right)\right)_{2-3 r_{0}} \rightarrow P$ defined by

- if $x \in U\left(C\left(I^{+}\right)\right)_{2-3 r_{0}}$, then $G(x)=g\left(I^{+}\right)(x)$,

- if $\left.\mathbf{v}_{c} \in E\left(S^{I}\right)\right|_{S^{I} \backslash \operatorname{Int}\left(U\left(C\left(I^{+}\right)\right)_{2-4 r_{0}}\right)}$, then $G\left(\mathbf{v}_{c}\right)=g_{0}\left(\mathbf{v}_{c}\right)$,

- if $\left.\mathbf{v}_{c} \in E\left(S^{I}\right)\right|_{S^{I} \cap U\left(C\left(I^{+}\right)\right)_{2-4 r_{0}}}$, then we define $G\left(\mathbf{v}_{c}\right)$ to be

$$
\exp _{P, g_{0}(c)}\left((1-\eta(c)) \exp _{P, g_{0}(c)}^{-1}\left(g\left(I^{+}\right)\left(\mathbf{v}_{c}\right)\right)+\eta(c) \exp _{P, g_{0}(c)}^{-1}\left(g_{0}\left(\mathbf{v}_{c}\right)\right)\right)
$$

where $\delta$ is so small that $E\left(S^{I}\right) \cap U\left(C\left(I^{+}\right)\right)_{2-3 r_{0}} \subset \pi_{E}^{-1}\left(S^{I} \cap U\left(C\left(I^{+}\right)\right)_{2-(7 / 2) r_{0}}\right)$ holds. It follows from Lemma 5.3 and Lemma 6.1 below that $G$ is an $\Omega$-regular map defined on $E\left(S^{I}\right) \cup U\left(C\left(I^{+}\right)\right)_{2-3 r_{0}}$, that $G \mid E\left(S^{I}\right)$ has the singularities of the symbol $I$ exactly on $S^{I}$, and that for any $c \in S^{I}$ and $1 \leq j \leq k+1$,

(1) $G\left|S^{I}=g_{0}\right| S^{I}=\pi_{P}^{\infty} \circ \mu \mid S^{I}$ and $(d G)_{c}=\left(d g_{0}\right)_{c}$,

(2) $j^{\infty} G$ is transverse to $\Sigma^{I}(N, P)$ and $S^{I}\left(j^{\infty} G\right)=S^{I}\left(j^{\infty} g_{0}\right)=S^{I}$,

(3) $Q\left(S^{I}\left(j^{\infty} G\right)\right)=Q\left(S^{I}\left(j^{\infty} g_{0}\right)\right)=Q, K_{j}\left(S^{I}\left(j^{\infty} G\right)\right)=K_{j}\left(S^{I}\left(j^{\infty} g_{0}\right)\right)=K_{j}$, $P_{j}\left(S^{I}\left(j^{\infty} G\right)\right)=P_{j}\left(S^{I}\left(j^{\infty} g_{0}\right)\right)=P_{j}$ and $T_{c}\left(S^{I_{j-1}}\left(j^{\infty} G\right)\right)=T_{c}\left(S^{I_{j-1}}\left(j^{\infty} g_{0}\right)\right)=$ $T_{c}\left(S^{I_{j-1}}(\mu)\right)$, 
(4) the following three homomorphisms are equal:

$$
\begin{aligned}
& \left(j^{\infty} G \mid S^{I}\right)^{*}\left(\mathbf{d}_{j+1} \circ d\left(j^{\infty} G\right) \mid T_{c}\left(S^{I_{j-1}}\left(j^{\infty} G\right)\right)\right), \\
& \left(j^{\infty} g_{0} \mid S^{I}\right)^{*}\left(\mathbf{d}_{j+1} \circ d\left(j^{\infty} g_{0}\right) \mid T_{c}\left(S^{I_{j-1}}\left(j^{\infty} g_{0}\right)\right)\right), \\
& \left(\mu \mid S^{I}\right)^{*}\left(\mathbf{d}_{j+1} \circ d \mu \mid T_{c}\left(S^{I_{j-1}}(\mu)\right)\right) .
\end{aligned}
$$

Under the identification $\mu^{*} \mathbf{P} \cong\left(\pi_{P}^{\infty} \circ \mu\right)^{*}(T P)$ and $\mu^{*} \mathbf{D} \cong T N$ in $(2.1)$, we have, in particular, that

$$
\left(j^{\infty} G \mid S^{I}\right)^{*}\left(\mathbf{d}_{j+1} \circ d\left(j^{\infty} G\right) \mid \mathfrak{n}\left(I_{j} \subset I_{j-1}\right)\right)=\left(\mu \mid S^{I}\right)^{*}\left(\mathbf{d}_{j+1} \circ d \mu \mid \mathfrak{n}\left(I_{j} \subset I_{j-1}\right)\right)
$$

over $S^{I}$. Set $\exp _{\Omega}=\exp _{\Omega(N, P)}$ for short. Let $h_{1}^{1}$ and $h_{0}^{3}$ be the maps $\left(E\left(S^{I}\right), S^{I}\right) \rightarrow$ $\left(\Omega(N, P), \Sigma^{I}(N, P)\right)$ defined by

$$
\begin{aligned}
& h_{1}^{1}\left(\mathbf{v}_{c}\right)=\exp _{\Omega, \mu(c)} \circ d_{c} \mu \circ\left(\exp _{N, c}\right)^{-1}\left(\mathbf{v}_{c}\right), \\
& h_{0}^{3}\left(\mathbf{v}_{c}\right)=\exp _{\Omega, j \infty G(c)} \circ d_{c}\left(j^{\infty} G\right) \circ\left(\exp _{N, c}\right)^{-1}\left(\mathbf{v}_{c}\right) \text {. }
\end{aligned}
$$

By applying Lemma 5.4 to the section $\mu$ and $h_{1}^{1}$, we first obtain a homotopy $h_{\lambda}^{1} \in$ $\Gamma_{\Omega}\left(E\left(S^{I}\right), P\right)$ between $h_{0}^{1}=\mu$ and $h_{1}^{1}$ on $E\left(S^{I}\right)$ satisfying properties $(5.4 .1),(5.4 .2)$ and (5.4.3) of Lemma 5.4. Similarly we obtain a homotopy $h_{\lambda}^{3} \in \Gamma_{\Omega}\left(E\left(S^{I}\right), P\right)$ between $h_{0}^{3}$ and $h_{1}^{3}=j^{\infty} G$ on $E\left(S^{I}\right)$ satisfying properties (5.4.1), (5.4.2) and (5.4.3).

Next we construct a homotopy of bundle maps $\mathfrak{n}(I) \rightarrow \nu\left(\Sigma^{I}\right)$ covering a homotopy $S^{I} \rightarrow \Sigma^{I}(N, P)$ between $d \mu \mid \mathfrak{n}(I)$ and $d\left(j^{\infty} G\right) \mid \mathfrak{n}(I)$. Let us recall the additive structure of $J^{\infty}(N, P)$ in $(1.2)$. Then we have the homotopy $\kappa_{\lambda}: S^{I} \rightarrow J^{\infty}(N, P)$ defined by

$$
\kappa_{\lambda}(c)=(1-\lambda) \mu(c)+\lambda j^{\infty} G(c) \quad \text { covering } \pi_{P}^{\infty} \circ \mu \mid S^{I}: S^{I} \rightarrow P,
$$

where $\pi_{P}^{\infty} \circ \mu \mid S^{I}$ is the immersion as in (5.2.3) of Lemma 5.2. We show that $\kappa_{\lambda}$ is actually a homotopy of $S^{I}$ to $\Sigma^{I}(N, P)$. Indeed, from the similar argument in the proof of Lemma 5.3 using (1), (2), (3) and (4) above it follows that the Boardman symbol of $\kappa_{\lambda}(c)$ is $I$ for any $\lambda$ and any $c \in S^{I}$ and that

$$
Q\left(S^{I}\left(\kappa_{\lambda}\right)\right)=Q, K_{j}\left(S^{I}\left(\kappa_{\lambda}\right)\right)=K_{j} \text { and } P_{j}\left(S^{I}\left(\kappa_{\lambda}\right)\right)=P_{j}
$$

Here, recall the direct sum decomposition

$$
\mathfrak{n}(\mu, I)=\bigoplus_{j=1}^{k} \mathfrak{n}\left(I_{j} \subset I_{j-1}\right)
$$

and the isomorphisms $\mathfrak{n}\left(I_{j} \subset I_{j-1}\right) \rightarrow P_{j}$. Then we can construct a splitting $i_{\lambda}^{\nu}: \nu\left(\Sigma^{I}\right) \rightarrow \mathbf{D}$ and direct sum decompositions

$$
\nu\left(\Sigma^{I}\right)=\bigoplus_{j=1}^{k} \nu\left(I_{j} \subset I_{j-1}\right) \text { and } \mathbf{K}_{1}=\bigoplus_{j=1}^{k-1}\left(\mathbf{K}_{j} / \mathbf{K}_{j+1}\right) \oplus \mathbf{K}_{k}
$$

over the images of $\kappa_{\lambda}\left(S^{I}\right)$ so that

$$
\begin{aligned}
d \mu\left(\mathfrak{n}\left(I_{j} \subset I_{j-1}\right)\right) & =i_{\lambda}^{\nu}\left(\left.\nu\left(I_{j} \subset I_{j-1}\right)\right|_{\mu\left(S^{I}\right)}\right), \\
d\left(j^{\infty} G\right)\left(\mathfrak{n}\left(I_{j} \subset I_{j-1}\right)\right) & =i_{\lambda}^{\nu}\left(\left.\nu\left(I_{j} \subset I_{j-1}\right)\right|_{j \infty G\left(S^{I}\right)}\right)
\end{aligned}
$$

and that these decompositions are compatible with $\mathbf{j}_{\mathbf{K}} \circ\left(\mu \mid S^{I}\right)^{\mathbf{K}_{1}}: K_{1}\left(S^{I}(\mu)\right) \rightarrow$ $\left.\mathbf{K}_{1}\right|_{\Sigma^{I}(N, P)} \rightarrow \nu\left(\Sigma^{I}\right)$. By the equalities of the homomorphisms in (6.6), we obtain a homotopy of bundle maps $\widetilde{\kappa_{\lambda}}: \mathfrak{n}(I) \rightarrow i_{\lambda}^{\nu}\left(\nu\left(\Sigma^{I}\right)\right)$ covering $\kappa_{\lambda}$ with the property 
(C) by using the homotopy $\left(\kappa_{\lambda}\right)^{i_{\lambda}^{\nu}\left(\nu\left(\Sigma^{I}\right)\right)}: \kappa_{\lambda}^{*}\left(i_{\lambda}^{\nu}\left(\nu\left(\Sigma^{I}\right)\right)\right) \rightarrow i_{\lambda}^{\nu}\left(\nu\left(\Sigma^{I}\right)\right)$ such that $\widetilde{\kappa_{0}}=d \mu \mid \mathfrak{n}(I)$ and $\widetilde{\kappa_{1}}=d\left(j^{\infty} G\right) \mid \mathfrak{n}(I)$. We define a homotopy $h_{\lambda}^{2}:\left(E\left(S^{I}\right), S^{I}\right) \rightarrow$ $\left(\Omega(N, P), \Sigma^{I}(N, P)\right)$ covering $\kappa_{\lambda}$ by

$$
h_{\lambda}^{2}\left(\mathbf{v}_{c}\right)=\exp _{\Omega, \mu(c)} \circ \widetilde{\kappa_{\lambda}} \circ\left(\exp _{N, c}\right)^{-1}\left(\mathbf{v}_{c}\right) .
$$

Then we have that $h_{0}^{2}\left(\mathbf{v}_{c}\right)=h_{1}^{1}\left(\mathbf{v}_{c}\right), h_{1}^{2}\left(\mathbf{v}_{c}\right)=h_{0}^{3}\left(\mathbf{v}_{c}\right)$ on $E\left(S^{I}\right)$. Since $h_{0}^{1}\left(\mathbf{v}_{c}\right)=$ $h_{1}^{3}\left(\mathbf{v}_{c}\right)=\mu\left(\mathbf{v}_{c}\right)$ for $\mathbf{v}_{c} \in U\left(C\left(I^{+}\right)\right)_{2-3 r_{0}}$, we may assume in the construction of $h_{\lambda}^{1}$, $h_{\lambda}^{2}$ and $h_{\lambda}^{3}$ that if $\mathbf{v}_{c} \in U\left(C\left(I^{+}\right)\right)_{2-3 r_{0}}$, then

$$
h_{\lambda}^{2}\left(\mathbf{v}_{c}\right)=h_{0}^{2}\left(\mathbf{v}_{c}\right)=h_{1}^{2}\left(\mathbf{v}_{c}\right) \text { and } h_{\lambda}^{1}\left(\mathbf{v}_{c}\right)=h_{1-\lambda}^{3}\left(\mathbf{v}_{c}\right) \text { for any } \lambda .
$$

Let $h_{\lambda}^{\prime} \in \Gamma_{\Omega}\left(E\left(S^{I}\right) \cup U\left(C\left(I^{+}\right)\right)_{2-3 r_{0}}, P\right)$ be the homotopy which is obtained by pasting $h_{\lambda}^{1}, h_{\lambda}^{2}$ and $h_{\lambda}^{3}$. The homotopies $h_{\lambda}^{1}$ and $h_{\lambda}^{3}$ are not homotopies relative to $E\left(S^{I}\right) \cap U\left(C\left(I^{+}\right)\right)_{2-3 r_{0}}$ in general. By using the above properties of $h_{\lambda}^{1}, h_{\lambda}^{2}$ and $h_{\lambda}^{3}$, we can modify $h_{\lambda}^{\prime}$ to a smooth homotopy $h_{\lambda} \in \Gamma_{\Omega}\left(E\left(S^{I}\right), P\right)$ satisfying the property $(\mathrm{C})$ such that

(1) $h_{\lambda}\left(\mathbf{v}_{c}\right)=h_{0}\left(\mathbf{v}_{c}\right)=\mu\left(\mathbf{v}_{c}\right)$ for any $\lambda$ and any $\mathbf{v}_{c} \in E\left(S^{I}\right) \cap U\left(C\left(I^{+}\right)\right)_{2-2 r_{0}}$,

(2) $h_{0}\left(\mathbf{v}_{c}\right)=\mu\left(\mathbf{v}_{c}\right)$ for any $\mathbf{v}_{c} \in E\left(S^{I}\right)$,

(3) $h_{1}\left(\mathbf{v}_{c}\right)=j^{\infty} G\left(\mathbf{v}_{c}\right)$ for any $\mathbf{v}_{c} \in E\left(S^{I}\right)$.

Since $S^{I} \backslash \operatorname{Int} U\left(C\left(I^{+}\right)\right)_{2-2 r_{0}}$ is closed and since $G$ is an $\Omega$-regular map such that $j^{\infty} G$ is transverse to $\Sigma^{I}(N, P)$, it follows from [G-G, Ch. II, Corollary 4.11] that there exists a homotopy $G_{\lambda}$ of $\Omega$-regular maps $E\left(S^{I}\right) \cup U\left(C\left(I^{+}\right)\right)_{2-2 r_{0}} \rightarrow P$ relative to $U\left(C\left(I^{+}\right)\right)_{2-2 r_{0}}$ with $G_{0}=G$ such that $j^{\infty} G_{\lambda}^{-1}\left(\bigcup_{J \geq I} \Sigma^{J}(N, P)\right)$ is contained in $\operatorname{Int}\left(\exp _{N}\left(D_{(1 / 2) \delta \circ \mu}(\mathfrak{n}(I))\right) \cup U\left(C\left(I^{+}\right)\right)_{2-2 r_{0}}\right)$, that $j^{\infty} G_{\lambda}$ is transverse to $\Sigma^{I}(N, P)$ for any $\lambda$ and that $j^{\infty} G_{1}$ is transverse to $\Sigma^{J}(N, P)$ for all symbols $J$. By using (1) and $G_{\lambda}$, we can extend $h_{\lambda}$ to the homotopy $H_{\lambda}^{\prime} \in \Gamma_{\Omega}\left(E\left(S^{I}\right) \cup U\left(C\left(I^{+}\right)\right)_{2-2 r_{0}}, P\right)$ defined by

$$
\begin{array}{ll}
H_{\lambda}^{\prime} \mid E\left(S^{I}\right)=h_{2 \lambda} & (0 \leq \lambda \leq 1 / 2), \\
H_{\lambda}^{\prime} \mid\left(E\left(S^{I}\right) \cup U\left(C\left(I^{+}\right)\right)_{2-2 r_{0}}\right)=j^{\infty} G_{2 \lambda-1} & (1 / 2 \leq \lambda \leq 1), \\
H_{\lambda}^{\prime}\left|U\left(C\left(I^{+}\right)\right)_{2-2 r_{0}}=\mu\right| U\left(C\left(I^{+}\right)\right)_{2-2 r_{0}} & (0 \leq \lambda \leq 1) .
\end{array}
$$

Furthermore, we slightly modify $H_{\lambda}^{\prime}$ to be smooth and replace $\delta$ and $E\left(S^{I}\right)$ by smaller ones. By the transversalities of $H_{\lambda}^{\prime}$ to $\Sigma^{I}(N, P)$ and of $H_{1}^{\prime}$ to $\Sigma^{J}(N, P)$ for all symbols $J$ and the homotopy extension property to $\mu$ and $H_{\lambda}^{\prime}$, we can extend $H_{\lambda}^{\prime}$ to a homotopy

$$
H_{\lambda}:\left(N, S^{I}\right) \rightarrow\left(\Omega(N, P), \Sigma^{I}(N, P)\right)
$$

relative to $U\left(C\left(I^{+}\right)\right)_{2-r_{0}}$ such that $H_{0}=\mu$ and $H_{1} \in \Gamma_{\Omega}^{t r}(N, P)$. Then $H_{\lambda}$ is the required homotopy in $\Gamma_{\Omega}(N, P)$ in the assertion $(\mathbf{A})$.

We finally prove the case $I=(n-p+1,0)$. Note that $\Omega^{I}(N, P)=\Sigma^{n-p}(N, P) \cup$ $\Sigma^{I}(N, P)$ and an $\Omega^{I}$-regular map is always transverse to $\Sigma^{I}(N, P)$. If we set $N_{0}=$ $S^{n-p}(s) \cup S^{I}(s)$, then $C\left(I^{+}\right)=C \cup\left(N \backslash N_{0}\right)$. Let us recall the neighborhoods $U\left(C\left(I^{+}\right)\right)^{\prime}$ and $U\left(C\left(I^{+}\right)\right)_{i}(i=1,2)$ of $C \cup\left(N \backslash N_{0}\right)$, where $g\left(I^{+}\right)$is defined. Since $s \in \Gamma_{\Omega^{L}}^{t r}(N, P), s \mid N_{0}$ is a section in $\Gamma_{\Omega^{I}}^{t r}\left(N_{0}, P\right)$ and $g\left(I^{+}\right) \mid\left(U\left(C\left(I^{+}\right)\right)^{\prime} \cap N_{0}\right)$ is an $\Omega^{I}$-regular map. Then it follows from [An4, Theorem 4.1] and [An6, Theorem 0.5] that there exist a homotopy $u_{\lambda} \in \Gamma_{\Omega^{I}}\left(N_{0}, P\right)$ relative to $U\left(C\left(I^{+}\right)\right)_{1} \cap N_{0}$ and an $\Omega^{I}$-regular map $f_{0}: N_{0} \rightarrow P$ such that $u_{0}=s \mid N_{0}$ and $u_{1}=j^{\infty} f_{0}$. The required homotopy $s_{\lambda}$ with an $\Omega^{L}$-regular map $g_{I}$ is defined by $s_{\lambda}\left|N_{0}=u_{\lambda}, s_{\lambda}\right| U\left(C\left(I^{+}\right)\right)_{2}=$ $j^{\infty} g\left(I^{+}\right)\left|U\left(C\left(I^{+}\right)\right)_{2}, g_{I}\right| N_{0}=f_{0}$ and $g_{I}\left|U\left(C\left(I^{+}\right)\right)_{2}=g\left(I^{+}\right)\right| U\left(C\left(I^{+}\right)\right)_{2}$. 
Lemma 6.1. Let $I, \mu, E\left(S^{I}\right)$ and $g_{0}$ be given as in the proof Theorem 4.1. Let the positive function $\delta$ in the definition of $E\left(S^{I}\right)$ be sufficiently small. Then we have, for every point $c \in S^{I}$,

(6.1.1) $\left(g_{0} \mid S^{I}\right)^{*}\left(d g_{0}\right)_{c}=d_{1}(\mu, I)_{c}$ and $Q\left(S^{I}\left(j^{\infty} g_{0}\right)\right)_{c}=Q\left(S^{I}(\mu)\right)_{c}$,

(6.1.2) for $1 \leq j \leq k+1$,

$\left(\mathrm{j}\right.$-a) $K_{j}\left(S^{I}\left(j^{\infty} g_{0}\right)\right)_{c}=K_{j, c}, P_{j}\left(S^{I}\left(j^{\infty} g_{0}\right)\right)_{c}=P_{j}\left(S^{I}(\mu)\right)_{c}$ and $T_{c}\left(S^{I_{j-1}}\left(j^{\infty} g_{0}\right)\right)$ $=T_{c}\left(S^{I_{j-1}}(\mu)\right)$,

(j-b) the following two homomorphisms to $Q\left(S^{I}(\mu)\right)_{c}$ are equal:

$$
\begin{aligned}
& \left(j^{\infty} g_{0} \mid S^{I}\right)^{*}\left(\mathbf{q}(k)^{j+1, j+1}\right) \mid\left(T_{c}\left(S^{I_{j-1}}(\mu)\right)\right) \bigcirc K_{j, c} \bigcirc K_{j-1, c} \bigcirc \cdots \circ K_{1, c}, \\
& \left(\mu \mid S^{I}\right)^{*}\left(\mathbf{q}(k)^{j+1, j+1}\right) \mid\left(T_{c}\left(S^{I_{j-1}}(\mu)\right)\right) \bigcirc K_{j, c} \bigcirc K_{j-1, c} \bigcirc \cdots \bigcirc K_{1, c},
\end{aligned}
$$

(j-c) the following two homomorphisms to $P_{j}\left(S^{I}(\mu)\right)_{c}$ are equal:

$$
\begin{aligned}
& \left(j^{\infty} g_{0} \mid S^{I}\right)^{*}\left(\mathbf{d}_{j+1} \circ d\left(j^{\infty} g_{0}\right) \mid\left(T_{c}\left(S^{I_{j-1}}(\mu)\right)\right)\right), \\
& \left(\mu \mid S^{I}\right)^{*}\left(\mathbf{d}_{j+1} \circ d \mu \mid\left(T_{c}\left(S^{I_{j-1}}(\mu)\right)\right)\right),
\end{aligned}
$$

(j-d) $T_{c}\left(S^{I_{j}}\left(j^{\infty} g_{0}\right)\right)=T_{c}\left(S^{I_{j}}(\mu)\right), \mathfrak{n}\left(j^{\infty} g_{0}, I_{j} \subset I_{j-1}\right)_{c}=\mathfrak{n}\left(\mu, I_{j} \subset I_{j-1}\right)_{c}$ and the sequence

$$
0 \longrightarrow T_{c}\left(S^{I_{j}}\left(j^{\infty} g_{0}\right)\right) \longrightarrow T_{c}\left(S^{I_{j-1}}(\mu)\right) \stackrel{h}{\longrightarrow} P_{j}\left(S^{I}(\mu)\right)_{c} \longrightarrow 0
$$

is exact, where $h=\left(\mu \mid S^{I}\right)^{*}\left(\mathbf{d}_{j+1} \circ d \mu \mid T_{c}\left(S^{I_{j-1}}(\mu)\right)\right)$.

Consequently, $j^{\infty} g_{0}$ is transverse to $\Sigma^{I_{j}}(N, P)$ along $S^{I}(\mu)$ and $g_{0}$ is an $\Omega$ regular map.

Proof. Let $K_{j}, Q$ and $P_{j}$ refer to $K_{j}\left(S^{I}(\mu)\right), Q\left(S^{I}(\mu)\right)$ and $P_{j}\left(S^{I}(\mu)\right)$ respectively. Let $c$ be a point of $S^{I}$. Since $\pi_{P}^{\infty} \circ \mu \mid S^{I}$ is an immersion and $\mathfrak{q}^{\prime}(\mu, I)^{1}=d_{1}(\mu, I) \mid \mathfrak{n}(I)$, we have $\left(g_{0} \mid S^{I}\right)^{*}\left(d g_{0}\right)_{c}=d_{1}(\mu, I)_{c}$. In particular, we have $K_{1}\left(S^{I}\left(j^{\infty} g_{0}\right)\right)=K_{1}$ and $Q\left(S^{I}\left(j^{\infty} g_{0}\right)\right)=Q$. We note that $d\left(\pi_{N}^{\infty}\right) \circ d\left(j^{\infty} g_{0}\right)_{c}=i d_{T_{c} N}$ and $d\left(\pi_{N}^{\infty}\right) \circ d(\mu)_{c}=$ $i d_{T_{c} N}$. Let us recall the notation $\mathfrak{c}_{\ell+1}\left(\mathfrak{s} \mid S^{I}\right)$ and $\mathfrak{b}_{\ell+1}\left(\mathfrak{s} \mid S^{I}\right)$ for $\mathfrak{s}=j^{\infty} g_{0}, \mu$ used in the proof of Lemma 5.3, which are induced from $\mathbf{c}_{j+1}$ with $\mathbf{P}$ being replaced by $\mathbf{Q}_{1}$ and $p_{\mathbf{Q}_{1}}^{\mathbf{p}} \circ \mathbf{b}_{\ell+1}$ respectively.

Let us prove Lemma 6.1 by induction on $j$. We may start with $j=0$. However, we begin by $j=1$ for the reader's convenience. We first have $P_{1}\left(S^{I}\left(j^{\infty} g_{0}\right)\right)_{c}=$ $P_{1, c}=\operatorname{Hom}\left(K_{1}, Q\right)_{c}$. It follows from (3.1), Lemma 3.1 and the definitions (6.4) and (6.5) for $g_{0}$ that for $\mathfrak{s}=j^{\infty} g_{0}, \mu$, the homomorphisms

$$
\left(\mathfrak{s} \mid S^{I}\right)^{*} \mathbf{q}(k)^{2,2} \mid \mathfrak{n}\left(\mu, I_{1} \subset I_{0}\right)_{c} \bigcirc K_{1, c}: \mathfrak{n}\left(\mu, I_{1} \subset I_{0}\right)_{c} \bigcirc K_{1, c} \longrightarrow Q_{c}
$$

coincide with each other and the homomorphisms $\left(\mathfrak{s} \mid S^{I}\right)^{*} \mathbf{q}(k)^{2,2} \mid T_{c}\left(S^{i_{1}}(\mathfrak{s})\right)_{c} \bigcirc K_{1, c}$ vanish. By the dimensional reason we have that

$$
\mathfrak{n}\left(j^{\infty} g_{0}, I_{1} \subset I_{0}\right)=\mathfrak{n}\left(\mu, I_{1} \subset I_{0}\right) \text { and } T_{c}\left(S^{i_{1}}\left(j^{\infty} g_{0}\right)\right)=T_{c}\left(S^{i_{1}}(\mu)\right) .
$$

Thus the equality in (j-c) for $j=1$ follows from the argument analogous to that in the proof of Lemma 5.3 using

$$
\mathfrak{c}_{1}\left(\mathfrak{s} \mid S^{I}\right) \circ \mathfrak{b}_{1}\left(\mathfrak{s} \mid S^{I}\right)=\left(\mathfrak{s} \mid S^{I}\right)^{*}\left(\mathbf{d}_{2} \circ d(\mathfrak{s}) \mid T_{c} N\right)
$$

for $\mathfrak{s}=j^{\infty} g_{0}, \mu$ in [B. Lemmas 7.11 and 7.13 and Definition 7.12] (by the definition of intrinsic derivatives, we may show the case $j=1$ by calculating the derivatives of the sections $\left(g_{0}\right)^{*}\left(d g_{0}\right)$ and $d_{1}(\mu, I)$ of $\operatorname{Hom}\left(T N,\left(\pi_{P}^{\infty} \circ \mu\right)^{*} T P\right)$ over

$$
\exp _{N, c}\left(\mathfrak{n}\left(\mu, I_{1} \subset I_{0}\right)_{c}\right)
$$


at $c)$. Furthermore, we have $T_{c}\left(S^{i_{1}}\left(j^{\infty} g_{0}\right)\right) \cap K_{1, c}=K_{2}\left(S^{I}\left(j^{\infty} g_{0}\right)\right)_{c}=K_{2, c}$.

Assume the assertions (6.1.2) for $j=\ell$. Then, we have

$$
K_{\ell+1}\left(S^{I}\left(j^{\infty} g_{0}\right)\right)_{c}=K_{\ell}\left(S^{I}\left(j^{\infty} g_{0}\right)\right)_{c} \cap T_{c}\left(S^{I_{\ell}}\left(j^{\infty} g_{0}\right)\right)=K_{\ell+1, c}
$$

and hence, $P_{\ell+1}\left(S^{I}\left(j^{\infty} g_{0}\right)\right)_{c}=P_{\ell+1, c}$ by (7) in Section 2. By (3.1), Lemma 3.1 and the definitions (6.4) and (6.5) for $g_{0}$, the two homomorphisms to $Q_{c}$

$$
\left(\mathfrak{s} \mid S^{I}\right)^{*} \mathbf{q}(k)^{\ell+2, \ell+2} \mid \mathfrak{n}\left(\mu, I_{\ell+1} \subset I_{\ell}\right)_{c} \bigcirc K_{\ell+1, c} \bigcirc K_{\ell, c} \bigcirc \cdots \bigcirc K_{1, c}
$$

for $\mathfrak{s}=j^{\infty} g_{0}, \mu$ coincide with each other. Furthermore, $\left(\mathfrak{s} \mid S^{I}\right)^{*} \mathbf{q}(k)^{\ell+2, \ell+2}$ vanish on $T_{c}\left(S^{I_{\ell+1}}(\mathfrak{s})\right) \bigcirc K_{\ell+1, c} \bigcirc K_{\ell, c} \bigcirc \cdots \bigcirc K_{1, c}$ for $\mathfrak{s}=j^{\infty} g_{0}, \mu$. By the dimensional reason we have that

$$
\mathfrak{n}\left(j^{\infty} g_{0}, I_{\ell+1} \subset I_{\ell}\right)=\mathfrak{n}\left(\mu, I_{\ell+1} \subset I_{\ell}\right) \text { and } T_{c}\left(S^{I_{\ell+1}}\left(j^{\infty} g_{0}\right)\right)=T_{c}\left(S^{I_{\ell+1}}(\mu)\right) .
$$

Hence, it follows that the two homomorphisms to $Q_{c}$

$$
\left(\mathfrak{s} \mid S^{I}\right)^{*} \mathbf{q}(k)^{\ell+2, \ell+2} \mid T_{c}\left(S^{I_{\ell}}(\mathfrak{s})\right) \bigcirc K_{\ell+1, c} \bigcirc K_{\ell, c} \bigcirc \cdots \bigcirc K_{1, c}
$$

for $\mathfrak{s}=j^{\infty} g_{0}, \mu$ coincide with each other. Thus the equality in (j-c) for $j=\ell+1$ follows from the equality $\mathfrak{c}_{\ell+1}\left(\mathfrak{s} \mid S^{I}\right) \circ \mathfrak{b}_{\ell+1}\left(\mathfrak{s} \mid S^{I}\right)=\left(\mathfrak{s} \mid S^{I}\right)^{*}\left(\mathbf{d}_{\ell+2} \circ d(\mathfrak{s}) \mid T_{c}\left(S^{I_{\ell}}(\mathfrak{s})\right)\right)$ for $\mathfrak{s}=j^{\infty} g_{0}, \mu$ in [B] Lemmas 7.11 and 7.13 and Definition 7.12]. Furthermore we have

$$
T_{c}\left(S^{I_{\ell+1}}\left(j^{\infty} g_{0}\right)\right) \cap K_{\ell+1}\left(S^{I}\left(j^{\infty} g_{0}\right)\right)_{c}=K_{\ell+2}\left(S^{I}\left(j^{\infty} g_{0}\right)\right)_{c}=K_{\ell+2, c}
$$

and the exact sequence $(6.9)$ for $\ell+1$.

Therefore, $j^{\infty} g_{0}$ is transverse to $\Sigma^{I_{\ell}}(N, P)$ along $S^{I}(\mu)$ for $1 \leq \ell \leq k+1$. Since $\Omega(N, P)$ is an open subset and $S^{I}\left(j^{\infty} g_{0}\right) \backslash \operatorname{Int} U\left(C\left(I^{+}\right)\right)_{2-3 r_{0}}$ is compact, we have that if $\delta$ is sufficiently small, then $j^{\infty} g_{0}\left(E\left(S^{I}\right)\right) \subset \Omega(N, P)$, namely, $g_{0}$ is an $\Omega$-regular map.

\section{Proof of Theorem 0.2}

In this section we prove Theorem 0.2 by applying Theorem 0.1 .

Proposition 7.1. Under the same assumptions as Theorem 0.2 , any section $s \in$ $\Gamma_{\Omega^{I}}^{t r}(N, P)$ has a homotopy $s_{\lambda} \in \Gamma_{\Omega^{I}}(N, P)$ such that $s_{0}=s$ and $s_{1} \in \Gamma_{\Omega^{J}}^{t r}(N, P)$.

We need the following lemma for the proof of Proposition 7.1. We provide $\mathbf{D}$ and $\mathbf{P}$ smooth metrics induced from Riemannian metrics on $N$ and $P$ respectively. Let us consider the splittings $\mathbf{K}_{j} \rightarrow \mathbf{K}_{r}(r \geq j)$ and $\mathbf{Q}_{1} \rightarrow \mathbf{P}$ to the inclusion $\mathbf{K}_{r} \rightarrow \mathbf{K}_{j}$ and the projection $\mathbf{P} \rightarrow \mathbf{Q}_{1}$ respectively. Then we may regard $\operatorname{Hom}\left(\bigcirc^{j} \mathbf{K}_{r}, \mathbf{Q}_{1}\right)$ as a subbundle of $\operatorname{Hom}\left(\mathbf{K}_{j} \bigcirc \mathbf{K}_{j-1} \bigcirc \cdots \bigcirc \mathbf{K}_{1}, \mathbf{P}\right)$ over $\Sigma^{I_{r}}(N, P)$.

Lemma 7.2. Assume the same assumptions as Theorem 0.2. Then, under the above remark, we have

(7.2.1) $\left.\mathbf{Q}_{1}\right|_{\Sigma^{I_{r}}(N, P)}$ and $\left.\bigcirc^{r+1} \mathbf{K}_{r}\right|_{\Sigma^{I_{r}}(N, P)}$ are trivial line bundles equipped with the canonical orientations respectively,

(7.2.2) the homomorphisms $\mathbf{c}_{j} \mid \operatorname{Hom}\left(\bigcirc^{j} \mathbf{K}_{r}, \mathbf{Q}_{1}\right)$ to $\mathbf{P}_{j}(1 \leq j \leq r)$ and $\mathbf{e}_{j} \circ$ $\mathbf{c}_{j-1} \mid \operatorname{Hom}\left(\bigcirc^{j-1} \mathbf{K}_{r}, \mathbf{Q}_{1}\right)$ to $\mathbf{Q}_{j}(1<j \leq r)$ are injective over $\Sigma^{I_{r}}(N, P)$.

Proof. In the proof $\Sigma^{I_{r}}$ refers to $\Sigma^{I_{r}}(N, P)$.

(7.2.1) By Section 2, property (5), $\mathbf{d}_{2} \mid \mathbf{K}_{1}: \mathbf{K}_{1} \rightarrow \operatorname{Hom}\left(\mathbf{K}_{1}, \mathbf{Q}_{1}\right)$ induces the isomorphism

$$
\mathbf{K}_{1} / \mathbf{K}_{2} \rightarrow \operatorname{Hom}\left(\mathbf{K}_{1} / \mathbf{K}_{2}, \mathbf{Q}_{1}\right) \quad \text { over } \Sigma^{I_{r}}
$$


This yields $\mathbf{q}: \mathbf{K}_{1} / \mathbf{K}_{2} \bigcirc \mathbf{K}_{1} / \mathbf{K}_{2} \rightarrow \mathbf{Q}_{1}$ over $\Sigma^{I_{r}}$, which is a nonsingular quadratic form on each fiber. Since $\operatorname{dim} \mathbf{K}_{1} / \mathbf{K}_{2}=n-p+1-i_{2}$ is odd, we choose the unique orientation of $\mathbf{Q}_{1}$, expressed by the unit vector $\mathbf{e}_{p}$, so that the index (the number of the negative eigenvalues) of $\mathbf{q}_{z}, z \in \Sigma^{I_{r}}$, is less than $\left(n-p+1-i_{2}\right) / 2$.

Since $\left.\mathbf{K}_{r}\right|_{\Sigma^{I_{r}}}$ is a line bundle and $r+1$ is even, $\left.\bigcirc^{r+1} \mathbf{K}_{r}\right|_{\Sigma^{I_{r}}}$ has the canonical orientation.

(7.2.2) We prove the assertion by induction on $j(r \geq 3)$. Since the kernel of $\mathbf{d}_{2} \mid \mathbf{K}_{1}$ is $\mathbf{K}_{2}$, we have that $\mathbf{c}_{1}=\mathbf{u}_{1}$ induces the inclusion $\operatorname{Hom}\left(\mathbf{K}_{r}, \mathbf{Q}_{1}\right) \rightarrow$ $\operatorname{Hom}\left(\mathbf{K}_{1}, \mathbf{Q}_{1}\right)=\mathbf{P}_{1}$ over $\Sigma^{I_{r}}$ and $\mathbf{e}_{2}: \mathbf{P}_{1} \rightarrow \mathbf{Q}_{2}$ is identified with the restriction $\operatorname{Hom}\left(\mathbf{K}_{1}, \mathbf{Q}_{1}\right) \rightarrow \operatorname{Hom}\left(\mathbf{K}_{2}, \mathbf{Q}_{1}\right)$ over $\Sigma^{I_{r}}$. Hence, $\mathbf{e}_{2} \circ \mathbf{c}_{1} \mid \operatorname{Hom}\left(\mathbf{K}_{r}, \mathbf{Q}_{1}\right)$ over $\Sigma^{I_{r}}$ is injective for $j=2$. Suppose that $\mathbf{e}_{j-1} \circ \mathbf{c}_{j-2} \mid \operatorname{Hom}\left(\bigcirc^{j-2} \mathbf{K}_{r}, \mathbf{Q}_{1}\right)$ is injective to $\mathbf{Q}_{j-1}$ over $\Sigma^{I_{r}}$ for $1 \leq j-2<r$. Then it follows from the definition of $\mathbf{u}_{j-1}$ in (2.6) that $\mathbf{c}_{j-1} \mid \operatorname{Hom}\left(\bigcirc^{j-1} \mathbf{K}_{r}, \mathbf{Q}_{1}\right)$ is injective to $\operatorname{Hom}\left(\mathbf{K}_{j-1}, \mathbf{Q}_{j-1}\right)$ over $\Sigma^{I_{r}}$. Since the image of $\mathbf{c}_{j-1}$ is $\mathbf{P}_{j-1}$, the map $\mathbf{c}_{j-1} \mid \operatorname{Hom}\left(\bigcirc^{j-1} \mathbf{K}_{r}, \mathbf{Q}_{1}\right)$ is injective to $\mathbf{P}_{j-1}$ over $\Sigma^{I_{r}}$. We next show that

$$
\mathbf{c}_{j-1}\left(\operatorname{Hom}\left(\bigcirc^{j-1} \mathbf{K}_{r}, \mathbf{Q}_{1}\right)\right) \cap \operatorname{Ker}\left(\mathbf{e}_{j}\right)=\{0\} .
$$

In the proof we use the notation $\mathbf{b}_{j-1}^{\prime}$ introduced in $\underline{B}$, (7.6)]. Recall that

$$
\operatorname{Ker}\left(\mathbf{e}_{j}\right)=\operatorname{Im}\left(\mathbf{c}_{j-1} \circ \mathbf{b}_{j-1}^{\prime} \mid \mathbf{K}_{j-1}\right) \text { and } \mathbf{d}_{j}=\mathbf{c}_{j-1} \circ \mathbf{b}_{j-1}^{\prime}
$$

in [B, Lemma 7.11]. Since $\mathbf{d}_{j} \mid \mathbf{K}_{j}$ vanishes and $\mathbf{K}_{r} \subset \mathbf{K}_{j}$ over $\Sigma^{I_{r}}$ and since $\mathbf{d}_{j}$ is symmetric, it is impossible that

$$
\mathbf{c}_{j-1}\left(\operatorname{Hom}\left(\bigcirc^{j-1} \mathbf{K}_{r}, \mathbf{Q}_{1}\right)\right) \subset \operatorname{Im}\left(\mathbf{d}_{j} \mid \mathbf{K}_{j-1}\right)=\operatorname{Im}\left(\mathbf{c}_{j-1} \circ \mathbf{b}_{j-1}^{\prime} \mid \mathbf{K}_{j-1}\right)=\operatorname{Ker}\left(\mathbf{e}_{j}\right) .
$$

Hence, the composition $\mathbf{e}_{j} \circ \mathbf{c}_{j-1} \mid \operatorname{Hom}\left(\bigcirc^{j-1} \mathbf{K}_{r}, \mathbf{Q}_{1}\right)$ is injective to $\mathbf{Q}_{j}$ over $\Sigma^{I_{r}}$ for $j \leq r$ by (5) in Section 2. Thus the map $\mathbf{c}_{j} \mid \operatorname{Hom}\left(\bigcirc^{j} \mathbf{K}_{r}, \mathbf{Q}_{1}\right)$ is injective to $\mathbf{P}_{j}$ over $\Sigma^{I_{r}}$ for $j \leq r$. This proves the lemma.

Proof of Proposition 7.1. In the proof we identify $J^{k}(N, P)$ with $J^{k}(T N, T P)$ by (1.2). By (9) in Section 2, there exist open subbundles $\widetilde{\Omega}^{L}(N, P)$ of $J^{k}(N, P)$ such that $\left(\pi_{k}^{\infty}\right)^{-1}\left(\widetilde{\Omega}^{L}(N, P)\right)=\Omega^{L}(N, P)$ for $L$ with length $k$. It follows that $\left(\pi_{r}^{\infty} \circ s\right)\left(N \backslash\left(S^{I_{r}}(s)\right)\right) \subset \widetilde{\Omega}^{I_{r-1}, 0}\left(N \backslash\left(S^{I_{r}}(s)\right), P\right)$.

We now construct a new section $\widetilde{u}: N \rightarrow \widetilde{\Omega}^{J}(N, P)$ as follows.

Let $\mathbf{e}_{p}\left(Q_{c}\right)$ and $\mathbf{e}\left(\bigcirc^{r+1}\left(K_{r}\right)_{c}\right)$ be the oriented unit vectors induced from the orientations of $\mathbf{Q}_{1, s(c)}$ and $\bigcirc^{r+1} \mathbf{K}_{r, s(c)}$ in Lemma 7.2 by $s$ respectively. Then we define the section $\phi^{J}: S^{I_{r}}(s) \rightarrow \operatorname{Hom}\left(\bigcirc^{r+1} K_{r}, Q\right)$ by $\phi^{J}(c)\left(\mathbf{e}\left(\bigcirc^{r+1}\left(K_{r}\right)_{c}\right)\right)=$ $\mathbf{e}_{p}\left(Q_{c}\right)$. Extend $\phi^{J}$ to a section $u_{\phi}: S^{I_{r}}(s) \rightarrow \operatorname{Hom}\left(S^{r+1}(T N),\left(\pi_{P}^{\infty} \circ s\right)^{*}(T P)\right)$ so that $u_{\phi}(c) \mid \bigcirc^{r+1} K_{r, c}=\phi^{J}(c)$ for $c \in S^{I_{r}}(s)$. Since $S^{I_{r}}(s)$ is a closed submanifold and since $\operatorname{Hom}\left(S^{r+1}(T N),\left(\pi_{P}^{\infty} \circ s\right)^{*}(T P)\right)$ is a vector bundle, we extend $u_{\phi}$ arbitrarily to the section $u_{\phi}^{N}: N \rightarrow \operatorname{Hom}\left(S^{r+1}(T N),\left(\pi_{P}^{\infty} \circ s\right)^{*}(T P)\right)$. Set $\widetilde{u_{\phi}}=\left(i d_{N} \times \pi_{P}^{\infty} \circ s\right)^{\mathbf{H}} \circ u_{\phi}^{N}$, where $\mathbf{H}=\operatorname{Hom}\left(S^{r+1}\left(\pi_{N}^{*}(T N)\right), \pi_{P}^{*}(T P)\right)$. Then we define $\widetilde{u}$ by $\widetilde{u}=\pi_{r}^{\infty} \circ s \oplus \widetilde{u_{\phi}}$ as the section of $J^{r+1}(N, P)=J^{r+1}(T N, T P)$. We lift $\widetilde{u}$ to the section $s^{J}$ of $J^{\infty}(N, P)$ over $N$. Then we have that $\pi_{r+1}^{\infty} \circ s^{J}=\widetilde{u}$ and $\pi_{r}^{\infty} \circ s^{J}=\pi_{r}^{\infty} \circ s$. Furthermore, we define the homotopy $s_{\lambda} \in \Gamma_{\Omega^{I}}(N, P)$ by

$$
s_{\lambda}=(1-\lambda) s+\lambda s^{J} .
$$

It follows from $\pi_{r}^{\infty} \circ s_{\lambda}=\pi_{r}^{\infty} \circ s=\pi_{r}^{\infty} \circ s^{J}$ that $s_{\lambda}$ is transverse to $\Sigma^{I_{r}}(N, P)$ and $S^{I_{r}}\left(s_{\lambda}\right)=S^{I_{r}}\left(s^{J}\right)=S^{I_{r}}(s)$. 
We prove that $s^{J} \in \Omega^{J}(N, P)$. For any point $c \in S^{I_{r}}(s)$, let $U_{c}$ be a convex neighborhood of $c$ and let $k$ and $y_{p}$ be the coordinates of $\exp _{N, c}\left(\left(K_{r}\right)_{c}\right)$ and $\exp _{P, \pi_{P}^{\infty} \circ s^{J}(c)}\left(\left(\pi_{P}^{\infty} \circ s^{J}\right)^{T P}(Q)_{c}\right)$ respectively. Let $D_{k}$ denote the vector of the total tangent bundle $\mathbf{D}$ which corresponds to $k$ as defined in $[\mathrm{B}$, Definition 1.6]. It follows from the definition of $\mathbf{D}$ that

$$
\left.\left(\bigcirc^{r+1} D_{k}\right) y_{p}\right|_{s^{J}(c)}=\partial^{r+1} y_{p} / \partial k^{r+1}(c) \neq 0 \quad \text { for } c \in S^{I_{r}}(s) .
$$

Then it follows from Lemma 7.2, (7.2.2), and $\mathbf{d}_{r+1}=\mathbf{c}_{r} \circ \mathbf{b}_{r}^{\prime}$ that

$$
\mathbf{d}_{r+1, s^{J}(c)} \mid \mathbf{K}_{r, s^{J}(c)}: \mathbf{K}_{r, s^{J}(c)} \rightarrow \mathbf{P}_{r, s^{J}(c)} \supset \mathbf{c}_{r}\left(\operatorname{Hom}\left(\bigcirc^{r} \mathbf{K}_{r, s^{J}(c)}, \mathbf{Q}_{1, s^{J}(c)}\right)\right)
$$

is injective. Hence, we have that $s^{J}\left(S^{I_{r}}(s)\right) \subset \Sigma^{J}(N, P)$. Since $s^{J}\left(N \backslash\left(S^{I_{r}}(s)\right) \subset\right.$ $\Omega^{I_{r-1}, 0}(N, P)$, the assertion is proved. Since we can deform $s^{J}$ to be transverse to $\Sigma^{L}(N, P)$ for all symbols $L$, we obtain a required homotopy $s_{\lambda}$.

Proof of Theorem 0.2. By assumption, we may assume that $j^{\infty} f$ is the section $N \rightarrow \Omega^{I}(N, P)$ which is transverse to $\Sigma^{L}(N, P)$ for all symbols $L$. By Proposition 7.1, we have a section $s^{J}: N \rightarrow \Omega^{J}(N, P)$ such that $j^{\infty} f$ and $s^{J}$ are homotopic in $\Gamma_{\Omega^{I}}(N, P)$. By Theorem 0.1 we obtain an $\Omega^{J}$-regular map $g$ such that $j^{\infty} g$ and $s^{J}$ are homotopic in $\Gamma_{\Omega^{I}}(N, P)$. This proves the assertion.

Corollary 7.3. Let $n \geq p \geq 2$, and let $N$ and $P$ be as above. Let $I=(n-p+1$, $1,1,1)$ and $J=(n-p+1,1,1,0)$ such that $n-p$ is an odd integer. Then if $f: N \rightarrow P$ is an $\Omega^{I}$-regular map, then $f$ is homotopic to an $\Omega^{J}$-regular map $g: N \rightarrow P$ such that $j^{\infty} f$ and $j^{\infty} g$ are homotopic in $\Gamma_{\Omega^{I}}(N, P)$.

This corollary proves the Chess conjecture $([\mathrm{C}]$. Sadykov Sady has actually proved a similar assertion corresponding to $J=(n-p+1,1,0)$ in the case of $N$ and $P$ being orientable.

Let $\pi_{0}(X)$ be the arcwise connected components of $X$. Theorem 0.1 asserts that

$$
\left(j_{\Omega^{I}}\right)_{*}: \pi_{0}\left(C_{\Omega^{I}}^{\infty}(N, P)\right) \rightarrow \pi_{0}\left(\Gamma_{\Omega^{I}}(N, P)\right)
$$

is surjective. However, $\left(j_{\Omega^{I}}\right)_{*}$ is not necessarily injective. Let $N=S^{2}, P=\mathbb{R}^{2}$ and $I=(1,0)$. Then we have by [An3 that $\Omega^{1,0}(2,2)$ is homotopy equivalent to $S O(3)$. It follows from [Ste, 36.4] that every two sections of $\Omega^{1,0}\left(S^{2}, \mathbb{R}^{2}\right)$ over $S^{2}$ are mutually homotopic. Namely, $\pi_{0}\left(\Gamma_{\Omega^{I}}(N, P)\right)$ consists of a single element. On the other hand, let $f_{\lambda}: S^{2} \rightarrow \mathbb{R}^{2}$ be a homotopy of fold-maps. Define $F: S^{2} \times[0,1] \rightarrow$ $\mathbb{R}^{2}$ by $F(x, \lambda)=f_{\lambda}(x)$ so that if $\lambda$ is sufficiently small, then $F(x, \lambda)=f_{0}(x)$ and $F(x, 1-\lambda)=f_{1}(x)$. By a very small perturbation of $F$ fixing $f_{0}$ and $f_{1}$, we may assume that $F$ is smooth and $f_{\lambda}$ is still an $\Omega^{1,0}$-regular map for any $\lambda$. Furthermore, the map $F: S^{2} \times[0,1] \rightarrow \mathbb{R}^{2} \times[0,1]$ becomes an $\Omega^{1,0}$-regular map, and $S^{1,0}(F)$ is a submanifold of $S^{2} \times[0,1]$. By the Jacobian matrix of $F$ we know that the kernel line bundle $K_{1}\left(j^{\infty} F\right)$ over $S^{1,0}(F)$ is independent with $\partial / \partial \lambda$, and $T\left(S^{1,0}(F)\right) \cap K_{1}\left(j^{\infty} F\right)=\{\mathbf{0}\}$. This implies that $S^{1,0}(F)$ is regularly projected onto $[0,1]$. Hence, $S^{1,0}\left(f_{0}\right)$ must be diffeomorphic to $S^{1,0}\left(f_{1}\right)$. Thus we conclude that $\pi_{0}\left(C_{\Omega^{I}}^{\infty}\left(S^{2}, \mathbb{R}^{2}\right)\right)$ is an infinite set.

\section{ACKNOWLEDGMENT}

The author would like to thank the referee for his/her careful reading of the manuscript and helpful comments. 


\section{REFERENCES}

[An1] Y. Ando, On the elimination of Morin singularities, J. Math. Soc. Japan 37(1985), 471487. MR0792988 (87h:58018)

[An2] Y. Ando, An existence theorem of foliations with singularities $A_{k}, D_{k}$ and $E_{k}$, Hokkaido Math. J. 19(1991), 571-578. MR1134991 (93c:58030)

[An3] Y. Ando, The homotopy type of the space consisting of regular jets and folding jets in $J^{2}(n, n)$, Japanese J. Math. 24(1998), 169-181. MR.1630137 (99e:58019)

[An4] Y. Ando, Fold-maps and the space of base point preserving maps of spheres, J. Math. Kyoto Univ. 41(2002), 691-735. MR1891672 (2003a:57060)

[An5] Y. Ando, Invariants of fold-maps via stable homotopy groups, Publ. RIMS, Kyoto Univ. 38(2002), 397-450. MR1903746 (2003f:57057)

[An6] Y. Ando, Existence theorems of fold-maps, Japanese J. Math. 30(2004), 29-73. MR2070370 (2005h:58070)

[An7] Y. Ando, The homotopy principle in the existence level for maps with only singularities of types $A, D$ and $E$, submitted to Nagoya Math. J. in 2003, http://front.math.ucdavis. edu/math.GT/0411399.

[An8] Y. Ando, Cobordisms of maps without prescribed singularities, http://front.math. ucdavis.edu/math.GT/0412234.

[B] J. M. Boardman, Singularities of differentiable maps, IHES Publ. Math. 33(1967), 21-57. MR 0231390 (37:6945)

[C] D. Chess, A note on the class $\left[S_{1}^{k}(f)\right]$, Proceedings of Symposia in Pure Math. 40(1983), Part 1, AMS, 221-224. MR0713061 (85f:57020)

[duP] A. du Plessis, Maps without certain singularities, Comment. Math. Helv. 50(1975), 363382. MR0397779 (53:1637)

[E1] J. M. Èliašberg, On singularities of folding type, Math. USSR. Izv. 4(1970), 1119-1134. MR.0278321 (43:4051)

[E2] J. M. Ėliašberg, Surgery of singularities of smooth mappings, Math. USSR. Izv. 6(1972), 1302-1326. MR0339261 (49:4021)

[F] S. Feit, $k$-mersions of manifolds, Acta Math. 122(1969), 173-195. MR0243541 (39:4862)

[G-G] M. Golubitsky and V. Guillemin, Stable Mappings and Their Singularities, SpringerVerlag, Berlin, Heidelberg, 1973. MR0341518(49:6269)

[G1] M. Gromov, Stable mappings of foliations into manifolds, Math. USSR. Izv. 3(1969), 671-694. MR0263103 (41:7708)

[G2] M. Gromov, Partial Differential Relations, Springer-Verlag, Berlin, Heidelberg, 1986. MR 0864505 (90a:58201)

[H1] M. Hirsch, Immersions of manifolds, Trans. Amer. Math. Soc. 93(1959), 242-276. MR.0119214(22:9980)

[H2] M. Hirsch, Differential Topology, Springer-Verlag, Berlin, Heidelberg, 1976. MR0448362 $(56: 6669)$

[K-N] S. Kobayashi and K. Nomizu, Foundations of Differential Geometry, Vol. 1, Interscience Publishers, New York, 1963. MR0152974 (27:2945)

[L1] H. I. Levine, Elimination of cusps, Topology 3(1965), 263-296. MR0176484 (31:756)

[L2] H. I. Levine, Singularities of differentiable maps, Proc. Liverpool Singularities Symposium, I, Springer Lecture Notes in Math. Vol. 192, Springer-Verlag, 1971, 1-85.

[Math1] J. N. Mather, Stability of $C^{\infty}$ mappings, IV: Classification of stable germs by $\mathbb{R}$-algebra, Publ. Math. Inst. Hautes Étud. Sci. 37(1970), 223-248. MR0275460 (43:1215b)

[Math2] J. N. Mather, On Thom-Boardman singularities, Dynamical Systems, Academic Press, 1973, 233-248. MR 0353359 (50:5843)

[Mats] Y. Matsushima, Differentiable Manifolds, Marcel Dekker, New York, 1972. MR0346831 (49:11553)

[Mo] B. Morin, Formes canoniques des singularités d'une application différentiable, C. R. Acad. Sci. Paris 260(1960), 6503-6506. MR0190944 (32:8354)

[P] A. Phillips, Submersions of open manifolds, Topology 6(1967), 171-206. MR0208611 $(34: 8420)$

[Sady] R. Sadykov, The Chess conjecture, Algebr. Geom. Topol. 3(2003), 777-789. MR1997337 (2005a:57027) 
[Ste] N. Steenrod, The Topology of Fibre Bundles, Princeton Univ. Press, Princeton, 1951. MR0039258 (12:522b)

[T] R. Thom, Les singularités des applications différentiables, Ann. Inst. Fourier 6(1955-56), 43-87. MR0087149(19:310a)

Department of Mathematical Sciences, Faculty of Science, Yamaguchi University, YAMAGUCHI 753-8512, JAPAN

E-mail address: andoy@yamaguchi-u.ac.jp 